\title{
MORITA EQUIVALENCE FOR SEMIGROUPS
}

\author{
S. TALWAR \\ (Received 5 May 1992; revised 16 June 1993) \\ Communicated by P. G. Trotter
}

\begin{abstract}
In this paper we shall extend the classical theory of Morita equivalence to semigroups with local units. We shall use the concept of a Morita context to rediscover the Rees theorem and to characterise completely 0 -simple and regular bisimple semigroups.
\end{abstract}

1991 Mathematics subject classification (Amer. Math. Soc.): 20 M50.

\section{Introduction}

Category theory allows us to study simultaneously certain aspects of a large number of mathematical structures. A deep application of the theory is Morita's (1961) equivalence theory for categories R-Mod, of 'unital' modules over rings with identity, which gives a new insight into the classical Artin-Wedderburn structure theorem for simple rings. The theorem can be interpreted as asserting that a ring $R$ with identity is simple artinian if and only if the category R-Mod is 'the same' as the category D-Mod for some division ring $D$.

The principal questions considered in Morita theory are: When are the two categories R-Mod and S-Mod equivalent, and how are such equivalences realised?

Knauer (1972) and Banaschewski (1972), independently parallel the ring theoretic approach. The two authors present essentially the same results. Knauer considers a non-additive category of $A$-modules, that is, instead of a ring, he takes a monoid $A$ which acts on sets from the left. He calls the category defined by such objects A-Act. For a given monoid $A$ he describes all monoids $B$ such that the category B-Act is equivalent to the category A-Act. In particular, he finds that the equivalence of these categories yields an isomorphism between the monoids $A$ and $B$ if $A$ is a group or

(C) 1995 Australian Mathematical Society 0263-6115/95 \$A2.00+0.00 
finite or commutative. This differs from the additive case where the categories of modules over a commutative field and its ring of $n \times n$ matrices are equivalent.

Abrams (1983), made a first step in extending the theory to rings without identity. He considered rings in which a set of commuting idempotents is given such that every element of the ring admits one of these idempotents as a two-sided unit, and the categories of all left modules which are unitary in a natural sense. Anh and Marki (1987) extend this theory to cover a wider range of rings and transfer more of the classical Morita theory as presented in Anderson and Fuller (1974). Their rings require any two elements to have a common two-sided identity, a condition which is fulfilled by all regular rings (in the sense of von Neumann). Their framework yields a new proof of Jacobson's theorem on simple rings with minimal condition for principal one-sided ideals.

All the above treatments consider those modules which are unitary, that is, $R M=$ $M$ for $R$ a ring (monoid), and $M$ a left $R$-module (act). In all cases we find that there exists a functor $F$ defined on the category of all left $R$-modules (acts) such that $F$ is equivalent to the identity functor on the subcategory of unitary left $R$-modules (acts). We may think of the unitary subcategories as 'fixed objects' of the appropriate functors.

In extending the theory to semigroups without identity we use the above as our motivation. We show that there exists a functor $F$ defined on the category $\mathbf{S}$-Act such that $S$ is a fixed object of $F$ and that $F(S)$ is a semigroup isomorphic to $S$. In so doing we discover a representation, namely $S \otimes_{S} \operatorname{Hom}(S, S)$, of semigroups with local units (that is, semigroups in which every element of $S$ has an idempotent as a left unit and an idempotent as a right unit, a condition fulfilled by all regular semigroups). Moreover we find that our representation reduces to the Vagner-Preston representation in the inverse case.

Using the representation to define the functor $S \otimes_{S} \operatorname{Hom}(S,-)$ on the category S-Act we concentrate our attention on the subcategory FS-Act of fixed objects of this functor. We find that FS-Act is a subcategory of the category of unitary $S$-acts (US-Act). Furthermore all projectives in US-Act lie in FS-Act.

We then proceed to develop the necessary machinery to achieve our goals, namely, necessary and sufficient conditions for FS-Act and FR-Act to be equivalent, where $S$ and $R$ are semigroups with local units. By analogy with ring theory, we define a Morita context to be a six-tuple $\left\langle S, R,{ }_{s} P_{R},{ }_{R} Q_{S}, \tau, \mu\right)$, where ${ }_{S} P_{R}$ is an $S$ - $R$-biact, ${ }_{R} Q_{S}$ is an $R$-S-biact, $\tau$ is an $S$-S-morphism of $P \otimes Q$ into $S$ and $\mu$ is an $R$ - $R$-morphism of $Q \otimes P$ into $R$. Our main theorem states that FS-Act and FR-Act are equivalent if and only if there exists a Morita context with $\tau$ and $\mu$ surjective.

As an example we describe those semigroups which are Morita equivalent to a monoid. We find that such an exposition also leads us to a new proof of the Rees theorem for completely 0 -simple semigroups, furthering the list of analogies between 
simple rings with minimal one-sided ideals and completely 0 -simple semigroups. Moreover, we are able to characterise completely 0 -simple (bisimple with regular 0 ) semigroups as precisely those regular semigroups which are Morita equivalent to a group with zero (a bisimple monoid with 0 ).

\section{The categories S-Act and US-Act}

DEFINITION. Let $S$ be a semigroup. A set $M$ together with a function $S \times M \rightarrow M$, denoted $(s, x) \mapsto s x$, satisfying $(s t) x=s(t x)$ is called a (left) $S$-act.

For a semigroup $S$ with 0 we are concerned with pointed $S$-acts, where an $S$-act $M$ is pointed if it has a distinguished element, also denoted by 0 , such that $0 x=0 \in M$ for all $x \in M$. We note that for this element 0 in $M$ we have $s 0=0$ for all $s \in S$. We denote the category of pointed $S$-acts by $\mathbf{S}^{\mathbf{0}}$-Act.

Let $M$ and $N$ be two $S$-acts. A mapping $f: M \rightarrow N$ is called a (left) $S$-morphism if $(s x) f=s(x f)$. The left $S$-acts together with the $S$-morphisms form a category which we shall denote by S-Act.

As usual we shall denote a left $S$-act $M$ by ${ }_{S} M$ and the set of left $S$-morphisms from ${ }_{s} M$ into ${ }_{s} N$ by $\operatorname{Hom}_{s}(M, N)$. We note that $\operatorname{Hom}_{s}(M, M)$ (which we shall denote by End $\left._{S} M\right)$ is a monoid under the usual composition of maps.

Analogously, right $S$-acts are defined. Their category will be denoted by Act-S. If necessary we shall distinguish left and right $S$-acts by writing ${ }_{S} M$ and $M_{S}$ respectively.

We note that for a family $\left\{P_{i}: i \in I\right\}$ of $S$-acts, the product $\prod_{i \in I} P_{i}$ and the coproduct $\bigsqcup_{i \in I} P_{i}$ exist, being respectively isomorphic to the cartesian product and the disjoint union of the sets $P_{i}$ with a suitable action of $S$. If $S$ has a 0 and $\left\{P_{i}: i \in I\right\}$ is an indexed set of pointed $S$-acts, then their coproduct is the 0 -direct union of the $P_{i}$ which we denote by $\bigcup^{0} P_{i}$.

DEFINITION. If a set $M$ is simultaneously a left $S$-act and a right $R$-act such that $s(m r)=(s m) r$, for all $s \in S, m \in M, r \in R$, then we shall call $M$ an $S$ - $R$-biact and denote it by ${ }_{s} M_{R}$. If ${ }_{S} N_{R}$ is some other $S$-R -biact, then we say that a map $f: N \rightarrow M$ is an $S$-R-morphism if $f$ is simultaneously a left $S$-morphism and a right $R$-morphism.

We note that if $N$ is a left $S$-act, then it is naturally a right $\operatorname{End}_{S} N$-act. Moreover, if as usual we write morphisms to the right, then by definition of a left $S$-morphism we have: $s(n f)=(s n) f$ for all $s \in S, n \in N, f \in \operatorname{End}_{S} N$ and it follows that $N$ is an $S$-End $S N$-biact.

In what follows we shall be interested in certain subcategories of the category S-Act. 


\section{The category US-Act}

DEFINITION. We say that a semigroup $S$ has local units if for each $s \in S$ there exist idempotents (not necessarily unique) $e_{s},{ }_{s} f \in S$ such that $\left(e_{s}\right) s=s=s(s f)$. We call $e_{s}$ a left unit of $s$, and $s$ a right unit of $s$.

We note that all regular semigroups have local units as do all monoids.

DEFINITION. Let $S$ be a semigroup, and let $M$ be a left $S$-act. We say that $M$ is a unitary $S$-act if $S M=M$. If $S$ has local units then this implies that for each $m \in M$ there exists an idempotent $e_{m} \in S$ such that $e_{m} m=m$. The unitary left $S$-acts together with the $S$-morphisms form a full subcategory of $S$-Act, which we shall denote by US-Act. We shall sometimes refer to members of US-Act as $U S$-acts. Also, an $S$ - $R$-biact will be said to be unitary if it is a unitary left $S$-act and a unitary right $R$-act.

If $S$ is a monoid with identity 1 , then as in the case for rings with identity, we say that an $S$-act $M$ is unital if $1 m=m$ for all $m \in M$. Knauer (1972) requires this clause as part of his definition of an $S$-act. We note that if $S$ is a monoid, then the concepts of unital and unitary coincide.

Clearly, every semigroup with local units is a unitary $S$-act over itself, if we define an action by the multiplication of the semigroup. For an example of a non-unitary $S$-act, let $S$ be the semigroup of positive integers under addition. Then $S$ is a left $S$-act over itself. However, it is not unitary since the equation $1=x+y$ has no solutions in $S$.

DEFINITION. Let $M$ be an $S$-act. A subset $N \subseteq M$ is a subact of $M$ if $S N \subseteq N$.

If $S$ is a semigroup with local units and $M$ is any $S$-act, then the set

$$
S M=\{s m: s \in S, m \in M\}
$$

is clearly a unitary subact of $M$ which contains every unitary subact of $M$. For, if $N$ is a unitary subact of $M$, then $S N=N$ so that if $n \in N$, then $n=s p$ for some $s \in S, p \in N$ and hence $n \in S M$.

Generators. Generators play a central role in the study of equivalences between categories of acts over monoids as they do in categories of modules over rings with identity. To keep the discussion brief, we state Proposition 15.2 from Mitchell (1965), and take it as our definition.

PROPOSITION 1.1. If the category $C$ has coproducts, then an object $G$ of $C$ is a generator for $C$ if and only if for each $c \in \mathrm{obC}$ there is an epimorphism $\gamma$ : $\bigcup_{i \in I} G_{i} \rightarrow C$ for some set $I$, where each $G_{i}$ is isomorphic to $G$. 
Let $S$ be a semigroup with local units. Then the left $S$-act ${ }_{S} S$ is a generator for the category US-Act. For let ${ }_{s} M \in$ US-Act and for each $m \in M$, let $S_{m}$ be isomorphic to ${ }_{S} S$ via an isomorphism $\pi_{m}: S_{m} \rightarrow S$. Now define $\rho: \bigsqcup_{m \in M} S_{m} \rightarrow M$ by putting $s_{m} \rho=s m$; clearly $\rho$ is a surjective $S$-morphism.

DEFINITION. In the category S-Act we shall say that the family $\left\{G_{i}: G_{i} \in\right.$ S-Act, $i \in I$, is a family of generators for S-Act if every $M \in \mathbf{S}$-Act is an epimorphic image of a coproduct of isomorphic copies of $\bigsqcup_{i \in I} G_{i}$.

We note that if $S$ is a semigroup with local units then $\{S e: e \in E(S)\}$ where $E(S)=\left\{e \in S: e^{2}=e\right\}$ is a family of generators for US-Act.

It is a well-known fact that equivalences preserve generators and families of generators.

\section{Indecomposables and projectives in US-Act}

We now look at two concepts specifically in the category US-Act for $S$ a semigroup with local units. As in Knauer (1972) we shall describe all indecomposable projectives in US-Act in order to get a characterisation of all projectives in US-Act.

DEFINITION. The $S$-act $M$ is call decomposable if there are non-empty acts $M_{1}, M_{2}$ such that $M \cong M_{1} \amalg M_{2}$. Otherwise $M$ is called indecomposable.

We note that since coproducts are preserved by equivalences, so are indecomposables.

Throughout the following we shall assume that $S$ is a semigroup with local units unless otherwise stated.

LEMMA 2.1. A cyclic unitary $S$-act $P=S x, x \in P$ is indecomposable.

PROOF. If $S x=P_{1} \cup P_{2}$, then since there exists an idempotent $e_{x}$ in $S$ such that $\left(e_{x}\right) x=x$, we have that $x \in S x$. Let $x \in P_{1}$. Thus $P_{1}=S x$.

The existence of left units for $U S$-acts allows us to modify most of Knauer's results with relative ease; we shall therefore present them without proof. Moreover, we leave it as an exercise for the reader to prove the results when $S$ has a 0 .

LEMMA 2.2. Let $P_{i} \in \mathrm{US}$-Act, $i \in I$, be indecomposable subacts of $P \in U S$-Act, where $\bigcap_{i \in I} P_{i} \neq \emptyset$. Then $\bigcup_{i \in I} P_{i}$ is an indecomposable subact of $P$.

PROOF. See Lemma 2.2 of Knauer (1972). 
PROPOSITION 2.3. Every US-act $P$ is uniquely decomposable into a coproduct of indecomposable subacts $P_{i}$ of $P$ such that $P \cong \bigsqcup_{i \in I} P_{i}$.

PROOF. See Proposition 2.5 of Knauer (1972).

DEFINITION. $P \in \mathbf{S}$-Act is called projective if given any diagram

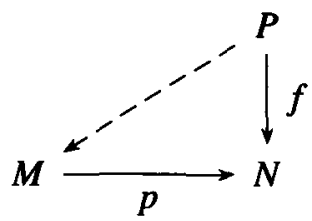

of $S$-acts and $S$-morphisms with $p$ surjective there exists an $S$-morphism $g: P \rightarrow M$ such that the triangle is commutative. In other words, given an epimorphism $p$ : $M \rightarrow N$, then any $S$-morphism $f: P \rightarrow N$ can be factored as $f=g p$ for some $g: P \rightarrow M$.

LEMMA 2.4. The left $S$-act $S e$, for e an idempotent of $S$, is projective.

PROOF. See Theorem 3.7 of Knauer (1972).

We note that it is clear that if $P \in \mathrm{US}-\mathrm{Act}$ and $\boldsymbol{P}$ is a projective in S-Act, then $P$ is also a projective in US-Act. Hence, for any idempotent $e$ in $S$ it follows from Lemma 2.4 that $S e$ is a projective in US-Act.

DEFINITION. A category has enough projectives if every object is an epimorphic image of a projective one.

LEMMA 2.5. The category US-Act has enough projectives.

PROOF. We have already noted that $\{S e: e \in E(S)\}$ is a family of generators for US-Act. Also it is a well-known fact that in any category the coproduct of projectives is projective. Thus the result follows from Lemma 2.4 and definition of a family of generators.

The next result is simply a restatement of Proposition II.14.2 of Mitchell (1965), in the special case of the category US-Act.

PROPOSITION 2.6. In the category US-Act the following are equivalent:

(1) The US-act $P$ is projective.

(2) Every epimorphism $M \rightarrow P, M \in \mathrm{US}$-Act, is a retract. 
Proposition 2.7. Let $P_{i} \in$ US-Act, $i \in I$. The US-act $\bigsqcup_{i \in I} P_{i}$ is projective if and only if $P_{i}$ is projective for every $i \in I$.

PROOF. See Proposition 3.3 of Knauer (1972).

Using Propositions 2.3 and 2.7 we shall now characterise all projectives in US-Act.

LEMMA 2.8. A projective $U S$-act $P$ is indecomposable if and only if $P$ is cyclic.

ProOF. See Lemma 3.5 of Knauer (1972).

Knauer (1972), makes the following remark: As there is no symmetric notion of an exact sequence in S-Act, Proposition 2.6 does not imply that a retract $f: M \rightarrow P$ onto a projective $P$ gives a decomposition of $M$, which is the case for modules.

THEOREM 2.9. $P$ is an indecomposable projective US-act if and only if $P \cong S e$ where $e$ is an idempotent of $S$.

PROOF. See Theorem 3.7 of Knauer (1972).

COROLlaRY 2.10. The $U S$-act $P$ is projective if and only if $P \cong \coprod_{i \in I} P_{i}$ for some set $I$ where for each $i$, there is an idempotent $e_{i}$ of $S$ such that $P_{i} \cong S e_{i}$.

PrOOF. The assertion follows from Propositions 2.3 and 2.7 and Theorem 2.9 above.

3. The Hom and $\otimes$ functors and a representation of semigroups with local units

As we shall see, homological algebra allows us to study a semigroup $S$ by investigating its category of left $S$-acts; this category, in turn, is investigated by examining the behaviour of certain functors on it, the most important of which are Hom, $\otimes$ and related functors derived from these.

The tensor product of acts. The tensor functor $\otimes$, arises from the concept of tensor product of left and right $S$-acts much in the same way as it does in the theory of modules over rings.

DEFINITION. For a right $S$-act $M_{S}$ and a left $S$-act ${ }_{S} N, M \otimes_{S} N$ is the solution of the usual universal problem: that is, $M \otimes{ }_{S} N=(M \times N) / \sigma$, where $\sigma$ is the equivalence relation on $M \times N$ generated by

$$
\Sigma=\{((x s, y),(x, s y)): x \in M, y \in N, s \in S\}
$$


We denote the $\sigma$-class of $(x, y)$ by $x \otimes y$.

As usual we refer to the set $M \otimes{ }_{s} N$ as the tensor product of $M_{S}$ and ${ }_{s} N$ and often write it as $M \otimes N$ when there is no ambiguity about the semigroup $S$. It follows from the universal property that up to isomorphism there is only one tensor product of $M_{S}$ and ${ }_{s} N$.

We often need to know when two elements $a \otimes b$ and $c \otimes d$ in a tensor product $M \otimes_{S} N$ are equal. From Proposition I.4.26 of Howie (1976), we know that $a \otimes b=c \otimes d$ if and only if either $(a, b)=(c, d)$, or for some positive integer $n \geq 2$ there is a sequence

$$
(a, b)=\left(a_{1}, b_{1}\right) \rightarrow\left(a_{2}, b_{2}\right) \rightarrow \ldots \rightarrow\left(a_{n}, b_{n}\right)=(c, d)
$$

in which for each $i \in\{1, \ldots, n-1\}$

either $\quad\left(\left(a_{i}, b_{i}\right),\left(a_{i+1}, b_{i+1}\right)\right) \in \Sigma \quad$ or $\quad\left(\left(a_{i+1}, b_{i+1}\right),\left(a_{i}, b_{i}\right)\right) \in \Sigma$.

Now $\left(\left(a_{i}, b_{i}\right),\left(a_{i+1}, b_{i+1}\right)\right) \in \Sigma$ means that for some $s_{i} \in S, a_{i}=a_{i+1} s_{i}, b_{i+1}=s_{i} b_{i}$. It follows immediately from this observation that if $M=S$ and $s \otimes b=t \otimes c$ in $S \otimes N$, then $s b=t c$.

We also note that if $P$ is a subact of $N$, it is clear that if $a, c \in M, b, d \in P$ and $a \otimes b=c \otimes d$ in $M \otimes P$, then we also have $a \otimes b=c \otimes d$ in $M \otimes N$. We caution that the converse of this assertion is not true in general. That is, it is possible to have $a \otimes b=c \otimes d$ in $M \otimes N$ but $a \otimes b \neq c \otimes d$ in $M \otimes P$ for appropriate choices of $M, N, P$.

Let $M$ be a fixed right $S$-act. Then we define the functor $\left(M \otimes_{S}-\right)$ by specifying that for any left $S$-act $N$ and any left $S$-morphism $g: N \rightarrow P$ of left $S$-acts we have

$$
\left(M \otimes_{S}-\right) N=M \otimes_{S} N \text { and }\left(M \otimes_{S}-\right)(g)=1_{M} \otimes g .
$$

Since $1_{M} \otimes g h=\left(1_{M} \otimes g\right)\left(1_{M} \otimes h\right)$ for $h: P \rightarrow Q$ and $1_{M} \otimes 1_{N}=1_{M \otimes N}$ we have that $\left(M \otimes_{S}-\right)$ is a functor from $S$-Act to Sets. Analogously we may fix a left $S$-act $N$ and define the functor $\left(-\otimes{ }_{s} N\right)$ from Act-S to Sets.

PROPOSITION 3.1. Let ${ }_{S} M_{R}$ be an $S$ - $R$-biact and ${ }_{R} N$ be an $R$-act. Then the tensor product ${ }_{S} M \otimes{ }_{R} N$ can be made into an $S$-act by defining $s(m \otimes n)=s m \otimes n$ for $s \in S, m \in M$ and $n \in N$.

Similarly, for a right $R$-act $M_{R}$ and an $R$ - $T$-biact ${ }_{R} N_{T}$ we get a right $T$-act $M \otimes_{R} N_{T}$ by defining $(m \otimes n) t=m \otimes n t$, and for biacts ${ }_{S} M_{R}$ and ${ }_{R} N_{T}$, these actions make ${ }_{S} M \otimes{ }_{R} N_{T}$ into an $S-T$-biact.

PROOF. That the actions are well-defined follows from our remarks on equality of elements in tensor products. The result is now clear. 
As a consequence of this proposition we may define the functor $\left(s M \otimes_{R}-\right)$ from R-Act to S-Act, for a fixed biact ${ }_{S} M_{R}$.

PROPOSITION 3.2. Let $S$ be a semigroup with local units and $M$ be a unitary $S$-act. Then $\kappa: S \otimes M \rightarrow M$ defined by $s \otimes m \mapsto s m$ is a surjective $S$-morphism.

PrOOF. That $\kappa$ is well-defined has been noted in our remarks on equality of elements in tensor products. It is clear that $\kappa$ is an $S$-morphism and surjectivity follows from the fact that $M$ is unitary.

The tensor product is an associative operation on acts. The following proposition is proved in exactly the same manner as is the module case in Jacobson (1980).

Proposition 3.3. For semigroups $R, S, T$ and $U$ we have an $R$ - $U$-biact isomorphism of $\left({ }_{R} M \otimes{ }_{S} N_{T}\right) \otimes_{T} P_{U}$ onto ${ }_{R} M_{S} \otimes\left({ }_{S} N \otimes_{T} P_{U}\right)$ given by $(x \otimes y) \otimes z \mapsto x \otimes(y \otimes z)$, for $x \in M, y \in N, z \in P$.

\section{The Hom acts and the Hom functor}

PROPOSITION 3.4. Let $R, S$ and $T$ be semigroups and ${ }_{S} N_{R}$ and ${ }_{S} U_{T}$ be biacts. Then $\operatorname{Hom}_{S}(U, N)$ is a left $T$-act and a right $R$-act if for $\Phi \in \operatorname{Hom}_{S}(U, N), r \in R$ and $t \in T$, we define the respective actions by

$$
\begin{array}{lll}
(t, \Phi) \mapsto t \cdot \Phi & \text { where } & t \cdot \Phi: u \in U \mapsto(u t) \Phi \in N \\
(\Phi, r) \mapsto \Phi \cdot r & \text { where } & \Phi \cdot r: u \in U \mapsto(u \Phi) r \in N .
\end{array} \quad \text { and }
$$

ProOF. Clearly $t \cdot \Phi \in \operatorname{Hom}_{S}(U, N)$, and the above map defines an action. Let $s, t \in T$ and $u \in U$. Then

$$
u(s \cdot(t \cdot \Phi))=(u s) t \cdot \Phi=(u s t) \Phi=u(s t \cdot \Phi) .
$$

If $R, S$ and $T$ are semigroups with local units and the biacts are unitary, then the subact

$$
T \operatorname{Hom}_{S}(U, N)=\left\{t \cdot \Phi: t \in T, \Phi \in \operatorname{Hom}_{S}(U, N)\right\}
$$

is the largest unitary $T$-subact of $\operatorname{Hom}_{S}(U, N)$.

By fixing $U$ we obtain the functor $T \operatorname{Hom}_{S}(U,-)$ which is induced by the mappings

$$
N \mapsto T \operatorname{Hom}_{S}(U, N) \quad \text { and } \quad f \in \operatorname{Hom}_{S}(N, M) \mapsto \bar{f}=T \operatorname{Hom}_{S}(U, f)
$$

where $\bar{f}: T \operatorname{Hom}_{S}(U, N) \rightarrow T \operatorname{Hom}_{S}(U, M)$ is defined by $t \cdot \Phi \mapsto(t \cdot \Phi) f$. 
We observe that $(t \cdot \Phi) f=t \cdot(\Phi f)$, for if $u \in U$, then

$$
u(t \cdot \Phi) f=(u(t \cdot \Phi)) f=((u t) \Phi) f=(u t)(\Phi f)=u(t \cdot(\Phi f)) .
$$

Similarly, the subact $\operatorname{Hom}_{S}(U, N) R$ is the largest right unitary $R$-act contained in $\operatorname{Hom}_{S}(U, N)$.

For a left $S$-act $N$ we have that $\operatorname{Hom}_{S}(S, N)$ is a left $S$-act. If $n$ is a fixed element of $N$ then the mapping $\rho(n): s \mapsto s n$ is a member of $\operatorname{Hom}_{S}(S, N)$. The morphism $t \cdot \Phi$ is closely related to the right multiplication map $\rho(t \Phi)$. In fact, $t \cdot \Phi=\rho(t \Phi)$, for if $s$ is an arbitrary element of $S$, then

$$
s(t \cdot \Phi)=(s t) \Phi=s(t \Phi)=s \rho(t \Phi) .
$$

Moreover, if $N=S$, then for all $s, t \in S$ we clearly have

$$
s \cdot \rho(t)=\rho(s t) .
$$

\section{The category FS-Act and a representation for semigroups with local units}

We now combine the Hom and tensor product functors to obtain a representation for semigroups with local units and to define a subcategory of US-Act for such semigroups. We begin by defining an important $S$-morphism as follows:

DEFINITION. For $M \in \mathbf{S}$-Act, define

$$
\Gamma_{M}: S \otimes{ }_{S} \operatorname{Hom}_{S}(S, M) \rightarrow M
$$

by putting

$$
(s \otimes \Phi) \Gamma_{M}=s \Phi
$$

LEMMA 4.1. The map $\Gamma_{M}$ is well-defined and is a morphism of left $S$-acts. Furthermore, if $M$ is a unitary $S$-act, then $\Gamma_{M}$ is surjective.

ProOF. Suppose that $s \otimes \Phi=t \Psi$ where $s, t \in S$ and $\Phi \Psi \in \operatorname{Hom}_{S}(S, M)$. Then from our comments on equality of elements in tensor products, we have that either $(s, \Phi)=(t, \Psi)$ in which case $s \Phi=t \Psi$ or for some $n \geq 2$, there is a sequence

$$
(s, \Phi)=\left(s_{1}, \Phi_{1}\right) \rightarrow\left(s_{2}, \Phi_{2}\right) \rightarrow \cdots \rightarrow\left(s_{n}, \Phi_{n}\right)=(t, \Psi)
$$

in which for each $i \in\{1, \ldots, n-1\}$ either

$$
\left(\left(s_{i}, \Phi_{i}\right),\left(s_{i+1}, \Phi_{i+1}\right)\right) \in \Sigma \quad \text { or } \quad\left(\left(s_{i+1}, \Phi_{i+1}\right),\left(s_{i}, \Phi_{i}\right)\right) \in \Sigma .
$$


If $\left(\left(s_{i}, \Phi_{i}\right),\left(s_{i+1}, \Phi_{i+1}\right)\right) \in \Sigma$, then for some $v_{i} \in S, s_{i}=s_{i+1} v_{i}$ and $\Phi_{i+1}=v_{i} \cdot \Phi_{i}$. Hence

$$
s_{i+1} \Phi_{i+1}=s_{i+1}\left(v_{i} \cdot \Phi_{i}\right)=\left(s_{i+1} v_{i}\right) \Phi_{i}=s_{i} \Phi_{i} .
$$

If $\left(\left(s_{i+1}, \Phi_{i+1}\right),\left(s_{i}, \Phi_{i}\right)\right) \in \Sigma$, then for some $v_{i} \in S, s_{i+1}=s_{i} v_{i}$ and $\Phi_{i}=v_{i} \cdot \Phi_{i+1}$. Hence

$$
s_{i+1} \Phi_{i+1}=\left(s_{i} v_{i}\right) \Phi_{i+1}=s_{i}\left(v_{i} \cdot \Phi_{i+1}\right)=s_{i} \Phi_{i}
$$

Thus

$$
s \Phi=s_{1} \Phi_{1}=s_{2} \Phi_{2}=\cdots=s_{n} \Phi_{n}=t \Psi,
$$

and $\Gamma_{M}$ is well-defined. It is clear that $\Gamma_{M}$ is an $S$-morphism.

Now suppose that $M$ is unitary and let $m \in M$. Then $m=s p$ for some $s \in S$ and $p \in M$. Then $\rho(p) \in \operatorname{Hom}_{S}(S, M)$ and

$$
(s \otimes \rho(p)) \Gamma_{M}=s \rho(p)=s p=m
$$

so that $\Gamma_{M}$ is surjective.

Let $M, N \in \mathrm{S}$-Act, and let $\Theta: M \rightarrow N$ be an $S$-morphism. We define

$$
\begin{gathered}
\Theta^{\dagger}=S \otimes{ }_{s} \operatorname{Hom}_{S}(S, \Theta): S \otimes{ }_{s} \operatorname{Hom}_{S}(S, M) \rightarrow S \otimes{ }_{s} \operatorname{Hom}_{s}(S, N), \\
\Theta^{\dagger}: s \otimes \Phi \mapsto s \otimes \Phi \Theta .
\end{gathered}
$$

$\Theta^{\dagger}$ is clearly well-defined and it is left as an easy exercise to show that $S \otimes_{S} \operatorname{Hom}_{S}(S,-)$ is a functor from $\mathbf{S}$-Act into $\mathbf{S}$-Act. In particular if $\Theta$ is an isomorphism then $\Theta^{\dagger}$ is an isomorphism. We now use this fact to define the category FS-Act.

Definition. We say that $M \in$ US-Act is a fixed object of the functor $S \otimes$ ${ }_{s} \operatorname{Hom}_{S}(S,-)$, if the $S$-morphism $\Gamma_{M}$ is an isomorphism. The class of fixed objects of $S \otimes{ }_{S} \mathrm{Hom}_{S}(S,-)$ together with the usual left $S$-morphisms defines a category which we shall denote by FS-Act. In view of Lemma 4.1, $\mathrm{M}$ is a fixed object if and only if $\Gamma_{M}$ is injective.

If $S$ has a 0 , then 0 is clearly a left $U S$-act. Moreover 0 is a fixed object. For if $s \otimes \Phi \in S \otimes{ }_{s} \operatorname{Hom}(S, 0)$, then letting $f$ be a left unit of $s$ we have that $s \otimes \Phi=$ $f \otimes s \cdot \Phi=f \otimes \rho(s \Phi)=0 \otimes \rho(0)$.

DEFINITION. Following ring and monoid theory we shall say that two semigroups $R$ and $S$ with local units are Morita equivalent if FS-Act is equivalent to FR-Act. If $R$ and $S$ both have a zero, then we shall require that $\mathbf{F S}^{\mathbf{0}}$-Act be equivalent to $\mathbf{F R}^{\mathbf{0}}$-Act. We note that FS-Act coincides with S-Act as studied by Knauer in the monoid case. 
Banaschewski (1972), makes the important observation that Morita equivalent semigroups (in the sense that R-Act and S-Act are equivalent, with no requirement that acts be unitary in any sense) are in fact isomorphic. So one is forced to define Morita equivalence in terms of subcategories if a notion differing from isomorphism is to be obtained. Knauer and Normak (1990), have shown that the monoids $A$ and $B$ are Morita equivalent if and only if ${ }_{A} C$ and ${ }_{B} C$ are equivalent categories, where ${ }_{A} C$ is the minimal full subcategory of A-Act which is closed under subacts, factor acts and contains $A$.

LEMMA 4.2. The restriction $S \otimes{ }_{S} \operatorname{Hom}_{S}(S,-)$ to FS-Act is naturally isomorphic to $1_{\mathrm{FS}-\mathrm{Act}}$.

PROOF. By the definition of $\Gamma_{M}$ we have that the following diagram

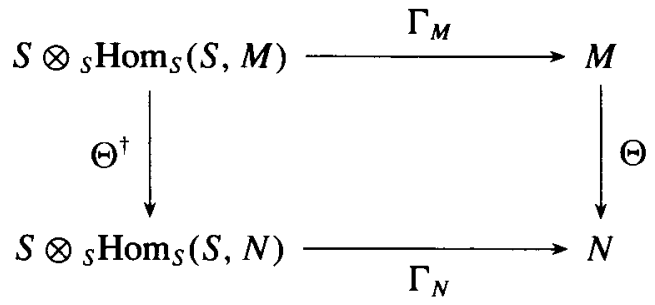

commutes. Since $\Gamma$ is an isomorphism by definition, the result follows.

We observe that the situation for a von-Neumann regular ring $R$ differs from ours in the sense that any two elements of $R$ lie in a subring of the form $e R e$, where $e$ is an idempotent of $R$. Thus the natural morphisms of $R \otimes M$ into $M$ and of $M$ into $R \operatorname{Hom}_{R}(R, M)$ are isomorphisms (see Anh and Marki (1987)). Since regular semigroups do not enjoy such a property we are led to consider more elaborate functors.

Proposition 4.3. Let $M=\bigcup\left\{M_{i}: i \in I\right\}$ be a unitary $S$-act where for each $M_{i}$ there is an idempotent $e_{i} \in S$ and an isomorphism $\Theta_{i}: M_{i} \rightarrow S e_{i}$. Then $\Gamma_{M}$ is an isomorphism, that is, $M \in$ FS-Act.

Proof. Let $s, t \in S, \Phi, \Psi \in \operatorname{Hom}_{S}(S, M)$ and suppose that $(s \otimes \Phi) \Gamma_{M}=(t \otimes$ $\Psi) \Gamma_{M}$, that is, $s \Phi=t \Psi$. Let $s \Phi \in M_{i}$. Then $s \Phi \Theta_{i}=t \Psi \Theta_{i} \in S e_{i}$.

Let $f^{2}=f \in S$ be such that $f\left(s \Phi \Theta_{i}\right)=s \Phi \Theta_{i}$ and $e_{s}^{2}=e_{s}, e_{t}^{2}=e_{t} \in S$ be such that $\left(e_{s}\right) s=s$ and $\left(e_{t}\right) t=t$. Then in $S \otimes \operatorname{Hom}_{s}\left(S, S e_{i}\right)$ we have $e_{s} \otimes \rho\left(s \Phi \Theta_{i}\right)=$ $e_{t} \otimes \rho\left(t \Psi \Theta_{i}\right)$. Hence $\left(e_{s} \otimes \rho(s \Phi)\right) \Theta_{i}^{\dagger}=\left(e_{t} \otimes \rho(t \Psi)\right) \Theta_{i}^{\dagger}$. Now $\Theta_{i}$ is an isomorphism and hence so is $\Theta_{i}^{\dagger}$. Thus $e_{s} \otimes \rho(s \Phi)=e_{t} \otimes \rho(t \Psi)$ in $S \otimes{ }_{S} \operatorname{Hom}_{S}\left(S, M_{i}\right)$. But $\operatorname{Hom}_{S}\left(S, M_{i}\right)$ is a subact of $\operatorname{Hom}_{S}(S, M)$ and so in view of our remarks about equality in tensor products we have $e_{s} \otimes \rho(s \Phi)=e_{t} \otimes \rho(t \Psi)$ in $S \otimes{ }_{s} \mathrm{Hom}_{S}(S, M)$. 
Now, in $S \otimes \operatorname{Hom}(S, M)$ we have

$$
s \otimes \Phi=e_{s} s \otimes \Phi=e_{s} \otimes s \cdot \Phi=e_{s} \otimes \rho(s \Phi)
$$

and similarly, $t \otimes \Psi=e_{t} \otimes \rho(t \Psi)$. Thus $s \otimes \Phi=t \otimes \Psi$ and $\Gamma_{M}$ is injective. In view of Lemma 4.1, we thus have that $\Gamma_{M}$ is an isomorphism.

COROLLARY 4.4. If $S$ is semigroup with local units, then ${ }_{S} S \cong S \otimes_{S} \operatorname{Hom}_{S}(S, S)$.

COROLlaRY 4.5. If $P \in$ US-Act is projective, then $P \in$ FS-Act.

PROOF. This is immediate from Proposition 4.3 and Corollary 2.10.

We remark that in the situation of Corollary 4.4 we can define a multiplication on $S \otimes \operatorname{Hom}_{S}(S, S)$ in such a way that $\Gamma_{S}$ becomes a semigroup isomorphism. To do this define

$$
(a \otimes \mu)(c \otimes \eta)=a \mu \otimes c \cdot \eta
$$

where $a, c \in S$ and $\mu, \eta \in \operatorname{Hom}_{S}(S, S)$. It is easy to prove directly that this definition gives a well-defined associative multiplication, and since

$$
\begin{aligned}
(a \mu \otimes c \cdot \eta) \Gamma_{s} & =(a \mu)(c \cdot \eta) \\
& =(a \mu) \rho(c \eta) \\
& =(a \mu)(c \eta) \\
& =(a \otimes \mu) \Gamma_{S}(c \otimes \eta) \Gamma_{S},
\end{aligned}
$$

we see that $\Gamma_{S}$ is a semigroup isomorphism.

The inverse of $\Gamma_{S}$ is the map $K$ given by $s \mapsto e \otimes \rho(s)$ where $s \in S$ and $e$ is an idempotent in $S$ such that $e s=s$. It is easy to see that the map is independent of the choice of the idempotent. For, if $f$ is some other idempotent in $S$ such that $f s=s$, then letting $g$ be a right unit of $s$, we have that

$$
e \otimes \rho(s)=e \otimes s \cdot \rho(g)=s \otimes \rho(g)=f \otimes s \cdot \rho(g)=f \otimes \rho(s) .
$$

Thus $K$ is a representation of a semigroup $S$ with local units. This representation can be regarded as a generalization of both the right regular representation of a monoid and the Vagner-Preston representation of an inverse semigroup (see Howie (1976)). For, if $S$ is a monoid, then there is an isomorphism of semigroups $\Omega: S \otimes \operatorname{Hom}_{S}(S, S) \rightarrow$ $\operatorname{Hom}_{S}(S, S)$ given by $s \otimes \Phi \mapsto s \cdot \Phi$. If $X: \operatorname{Hom}_{S}(S, S) \rightarrow T(S)$ is the inclusion map where $T(S)$ is the full transformation monoid on $S$, then the composed map $K \Omega X$ is the right regular representation of $S$. 
Now consider an inverse semigroup $S$ and a unitary $S$-act $M$. We claim that $B: S \otimes{ }_{S} M \rightarrow M$ given by $s \otimes m \mapsto s m$ is an isomorphism. We know that it is well-defined and it is clearly a surjective $S$-morphism. To see that it is injective, suppose that $s m=t n$ for some $s, t \in S$ and $m, n \in M$. Then

$$
t n=s m=s s^{-1} s m=s s^{-1} t n
$$

and similarly, $s m=t t^{-1} s m$. Hence

$$
\begin{aligned}
s \otimes m & =s s^{-1} s \otimes m=s s^{-1} \otimes s m=s s^{-1} \otimes t n \\
& =s s^{-1} t \otimes n=s s^{-1} t t^{-1} t \otimes n=t t^{-1} s s^{-1} t \otimes n \\
& =t t^{-1} \otimes s s^{-1} t n=t t^{-1} \otimes t n=t t^{-1} t \otimes n=t \otimes n .
\end{aligned}
$$

Now $\operatorname{Hom}_{S}(S, S)$ need not be unitary but $S \operatorname{Hom}_{S}(S, S)$ is unitary and we have

$$
S \otimes{ }_{S} \operatorname{Hom}_{S}(S, S)=S \otimes{ }_{S} S \operatorname{Hom}_{S}(S, S) .
$$

Indeed, more generally:

LEMMA 4.6. Let $R$ and $S$ be semigroups with local units, $U$ a unitary $R$ - $S$-biact and suppose $N$ is a left $R$-act. Then $S \otimes{ }_{S} \operatorname{Hom}_{R}(U, N)=S \otimes{ }_{S} S \operatorname{Hom}_{R}(U, N)$.

PROOF. It is clear that the natural map from $S \otimes S \operatorname{Hom}_{R}(U, N)$ to $S \otimes \operatorname{Hom}_{R}(U, N)$ is surjective. Let $s \otimes t \cdot \Phi=u \otimes v \cdot \eta$ in $S \otimes \operatorname{Hom}_{R}(U, N)$. Then either $(s, t$. $\Phi)=(u, v \cdot \eta)$, in which case $s \otimes t \cdot \Phi=u \otimes v \cdot \eta$ in $S \otimes S H \operatorname{Hom}(U, N)$, or for some $n \geq 2$ there exists a sequence, $(s, t \cdot \Phi)=\left(s_{1}, \Phi_{1}\right) \rightarrow\left(s_{2}, \Phi_{2}\right) \rightarrow \cdots \rightarrow$ $\left(s_{n}, \Phi_{n}\right)=(u, v \cdot \eta)$, in $S \times \operatorname{Hom}_{R}(U, N)$ such that either $\left(\left(s_{i}, \Phi_{i}\right),\left(s_{i+1}, \Phi_{i+1}\right)\right) \in \Sigma$, or $\left(\left(s_{i+1}, \Phi_{i+1}\right),\left(s_{i}, \Phi_{i}\right)\right) \in \Sigma$.

If $\left(\left(s_{i}, \Phi_{i}\right),\left(s_{i+1}, \Phi_{i+1}\right)\right) \in \Sigma$ then for some $x_{i} \in S, s_{i}=s_{i+1} x_{i}$ and $\Phi_{i+1}=x_{i} \cdot \Phi_{i}$. For each $i=1, \ldots, n$ let $f_{i}$ and $e_{i}$ be respectively a left and right unit of $s_{i}$. Then,

$$
\begin{aligned}
& \left(s_{i}, e_{i} \cdot \Phi_{i}\right)=\left(s_{i+1} x_{i}, e_{i} \cdot \Phi_{i}\right) \\
& \quad \rightarrow\left(f_{i+1}, s_{i+1} x_{i} e_{i} \cdot \Phi_{i}\right)=\left(f_{i+1}, s_{i} \cdot \Phi_{i}\right)=\left(f_{i+1}, s_{i+1} \cdot \Phi_{i+1}\right) \\
& \quad \rightarrow\left(s_{i+1}, e_{i+1} \cdot \Phi_{i+1}\right) .
\end{aligned}
$$

Now, the result follows from observing that each term in the above sequence lies in $S \times S \operatorname{Hom}_{R}(U, N)$.

Thus, for an inverse semigroup $S$ we have

$$
S \otimes{ }_{S} \operatorname{Hom}_{S}(S, S)=S \otimes{ }_{S} S \operatorname{Hom}_{S}(S, S) \cong S \operatorname{Hom}_{S}(S, S) .
$$

Consequently, we have 
COROLlaRY 4.7. If $S$ is an inverse semigroup, then $\kappa: S \rightarrow \operatorname{Hom}_{S}(S, S)$ given by $a \kappa=a a^{-1} \cdot \rho(a)$ is an isomorphism of semigroups.

Of course, $a a^{-1} \cdot \rho(a)=\rho\left(a a^{-1} a\right)=\rho(a)$ but we may also identify it with the partial one-one map with domain $\mathrm{Saa}^{-1}$ (see Howie (1976)). On doing this we recover the Vagner-Preston representation for an inverse semigroup.

We conclude this section by showing that the tensor product functor allows us to map US-Act into FS-Act. We shall use this fact to construct equivalence functors between the categories FS-Act and FR-Act. More specifically, we shall map a given $M \in$ FR-Act into US-Act and then map its image into FS-Act.

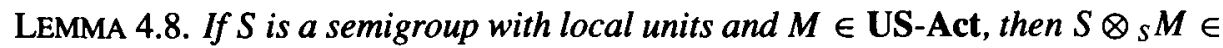
FS-Act.

PROOF. Define $K: S \otimes M \rightarrow S \otimes \operatorname{Hom}_{S}(S, S \otimes M)$ by $s \otimes m \mapsto s \otimes \rho\left(h_{m} \otimes m\right)$ where $h_{m}^{2}=h_{m} \in S$ and $h_{m} m=m$.

We note first that if $h$ is any idempotent in $S$ such that $h m=m$ then

$$
s \otimes \rho\left(h_{m} \otimes m\right)=s \otimes \rho(h \otimes m)
$$

For let $f^{2}=f \in S$ be such that $s f=s$. Then $s \otimes \rho\left(h_{m} \otimes m\right)=s \otimes \rho\left(h_{m} \otimes m\right)=$ $s \otimes f \cdot \rho\left(f h_{m} \otimes m\right)=s \otimes \rho(f \otimes m)=s \otimes \rho(f \otimes h m)=s \otimes f \cdot \rho(h \otimes m)=s \otimes \rho(h \otimes m)$.

Now let $s \otimes m=t \otimes p$ in $S \otimes M$. Then either $(s, m)=(t, p)$ in which case $s \otimes \rho\left(h_{m} \otimes m\right)=t \otimes \rho\left(h_{p} \otimes p\right)$ from above, or for some $n \geq 2$ there is a sequence

$$
(s, m)=\left(s_{1}, m_{1}\right) \rightarrow\left(s_{2}, m_{2}\right) \rightarrow \cdots \rightarrow\left(s_{n}, m_{n}\right)=(t, p)
$$

in which for every $i \in\{1, \ldots, n-1\}$ either

$$
\left(\left(s_{i}, m_{i}\right),\left(s_{i+1}, m_{i+1}\right)\right) \in \Sigma \quad \text { or } \quad\left(\left(s_{i+1}, m_{i+1}\right),\left(s_{i}, m_{i}\right)\right) \in \Sigma \text {. }
$$

If $\left(\left(s_{i}, m_{i}\right),\left(s_{i+1}, m_{i+1}\right)\right) \in \Sigma$, then for some $v_{i} \in S$,

$$
s_{i}=s_{i+1} v_{i} \quad \text { and } \quad m_{i+1}=v_{i} m_{i} .
$$

Now, $S$ is a semigroup with local units and $M$ is a unitary $S$-act, so we may assume that for each $i \in\{1, \ldots, n-1\}$ there exist idempotents $e_{i}, f_{i}, g_{i}, h_{i}$ in $S$ such that $e_{i} s_{i}=s_{i}=s_{i} f_{i}, v_{i} g_{i}=v_{i}$ and $h_{i} m_{i}=m_{i}$. Then, noting that $e_{i} s_{i+1} v_{i}=e_{i} s_{i}=s_{i}=$ $s_{i+1} v_{i}$ we have

$$
\begin{aligned}
s_{i} \otimes \rho\left(h_{i} \otimes m_{i}\right) & =e_{i} s_{i} \otimes \rho\left(h_{i} \otimes m_{i}\right) \\
& =e_{i} \otimes \rho\left(s_{i} h_{i} \otimes m_{i}\right) \\
& =e_{i} \otimes \rho\left(s_{i} \otimes m_{i}\right)
\end{aligned}
$$




$$
\begin{aligned}
& =e_{i} \otimes \rho\left(s_{i+1} v_{i} \otimes m_{i}\right) \\
& =e_{i} \otimes \rho\left(s_{i+1} v_{i} g_{i} \otimes m_{i}\right) \\
& =e_{i} \otimes s_{i+1} v_{i} \cdot \rho\left(g_{i} \otimes m_{i}\right) \\
& =e_{i} s_{i+1} v_{i} \otimes \rho\left(g_{i} \otimes m_{i}\right) \\
& =s_{i+1} v_{i} \otimes \rho\left(g_{i} \otimes m_{i}\right) \\
& =s_{i+1} f_{i+1} v_{i} \otimes \rho\left(g_{i} \otimes m_{i}\right) \\
& =s_{i+1} \otimes f_{i+1} v_{i} \cdot \rho\left(g_{i} \otimes m_{i}\right) \\
& =s_{i+1} \otimes \rho\left(f_{i+1} v_{i} g_{i} \otimes m_{i}\right) \\
& =s_{i+1} \otimes \rho\left(f_{i+1} \otimes v_{i} m_{i}\right) \\
& =s_{i+1} \otimes \rho\left(f_{i+1} \otimes m_{i+1}\right) \\
& =s_{i+1} \otimes \rho\left(f_{i+1} \otimes h_{i+1} m_{i+1}\right) \\
& =s_{i+1} \otimes f_{i+1} \cdot \rho\left(h_{i+1} \otimes m_{i+1}\right) \\
& =s_{i+1} f_{i+1} \otimes \rho\left(h_{i+1} \otimes m_{i+1}\right) \\
& =s_{i+1} \otimes \rho\left(h_{i+1} \otimes m_{i+1}\right)
\end{aligned}
$$

Similarly, if $\left(\left(s_{i+1}, m_{i+1}\right),\left(s_{i}, m_{i}\right)\right) \in \Sigma$, then

$$
s_{i+1} \otimes \rho\left(h_{i+1} \otimes m_{i+1}\right)=s_{i} \otimes \rho\left(h_{i} \otimes m_{i}\right)
$$

so that

$$
\begin{aligned}
s \otimes \rho\left(h_{m} \otimes m\right) & =s_{1} \otimes \rho\left(h_{1} \otimes m_{1}\right)=s_{2} \otimes \rho\left(h_{2} \otimes m_{2}\right)=\cdots \\
& =s_{n} \otimes \rho\left(h_{n} \otimes m_{n}\right)=t \otimes \rho\left(h_{p} \otimes p\right)
\end{aligned}
$$

and we have that $K$ is well-defined. $K$ is clearly a left $S$-morphism.

Noting that the map $\Gamma_{S \otimes M}: S \otimes \operatorname{Hom}_{S}(S, S \otimes M) \rightarrow S \otimes M$ is well-defined we can easily show that $K$ is injective. Suppose that $(s \otimes m) K=(t \otimes p) K$. Then $s \otimes \rho\left(h_{m} \otimes m\right)=t \otimes \rho\left(h_{p} \otimes p\right)$ so that $\left(s \otimes \rho\left(h_{m} \otimes m\right)\right) \Gamma_{S \otimes M}=\left(t \otimes \rho\left(h_{p} \otimes p\right)\right) \Gamma_{S \otimes M}$, that is, $s \otimes m=s\left(h_{m} \otimes m\right)=t\left(h_{p} \otimes p\right)=t \otimes p$.

To show that $K$ is surjective let $s \otimes \Phi \in S \otimes \operatorname{Hom}_{S}(S, S \otimes M)$. Then $s \Phi=a \otimes q$ for some $a \in S$ and $q \in M$. Also, $e s=s$ for some $e^{2}=e \in S$ and so $e(a \otimes q)=a \otimes q$. Now, $(a \otimes q) K=(e a \otimes q) K=e a \otimes \rho\left(h_{q} \otimes q\right)=e \otimes a \cdot \rho\left(h_{q} \otimes q\right)=e \otimes \rho(a \otimes q)=$ $e \otimes \rho(s \Phi)=e \otimes s \cdot \Phi=s \otimes \Phi$.

Furthermore, $\Gamma_{S \otimes M}$ is the inverse of $K$. For, let $s \otimes m \in S \otimes M$. Then

$$
(s \otimes m) K \Gamma_{S \otimes M}=\left(s \otimes \rho\left(h_{m} \otimes m\right)\right) \Gamma_{S \otimes M}=s\left(h_{m} \otimes m\right)=s \otimes m .
$$

That $\Gamma_{S \otimes M} K=1_{S \otimes M}$ follows from the proof of surjectivity. 


\section{The Morita equivalence}

We begin by reminding the reader that we shall say that two semigroups $R$ and $S$ with local units are Morita equivalent if FS-Act is equivalent to FR-Act.

Now we address our central question: When are two semigroups with local units equivalent, and how are the equivalences realised? The Hom and $\otimes$ functors will play a further role in our exposition, for we shall give the complete characterisations of equivalence in terms of them.

The central concept of our machinery is the classical notion of a Morita context (see Jacobson (1980)).

DEFINITION. A six-tuple $\left\langle R, S,{ }_{R} P_{S},{ }_{s} Q_{R}, \tau, \mu\right\rangle$ is said to be a Morita context, where $R$ and $S$ are semigroups, ${ }_{R} P_{S}$ is an $R$-S-biact, $s Q_{R}$ is an $S$-R-biact, $\tau$ is an $R$ - $R$-morphism of $P \otimes_{S} Q$ into $R$, and $\mu$ is an $S$-S-morphism of $Q \otimes_{R} P$ into $S$ such that if we put $(p \otimes q) \tau=\langle p, q\rangle$ and $(q \otimes p) \mu=[q, p]$, then for all $p, p^{\prime} \in P, q, q^{\prime} \in Q$, we have

(1) $\langle p, q\rangle p^{\prime}=p\left[q, p^{\prime}\right]$.

(2) $q\left\langle p, q^{\prime}\right\rangle=[q, p] q^{\prime}$.

The conditions 1 and 2 are equivalent respectively to the commutativity of the following diagrams.
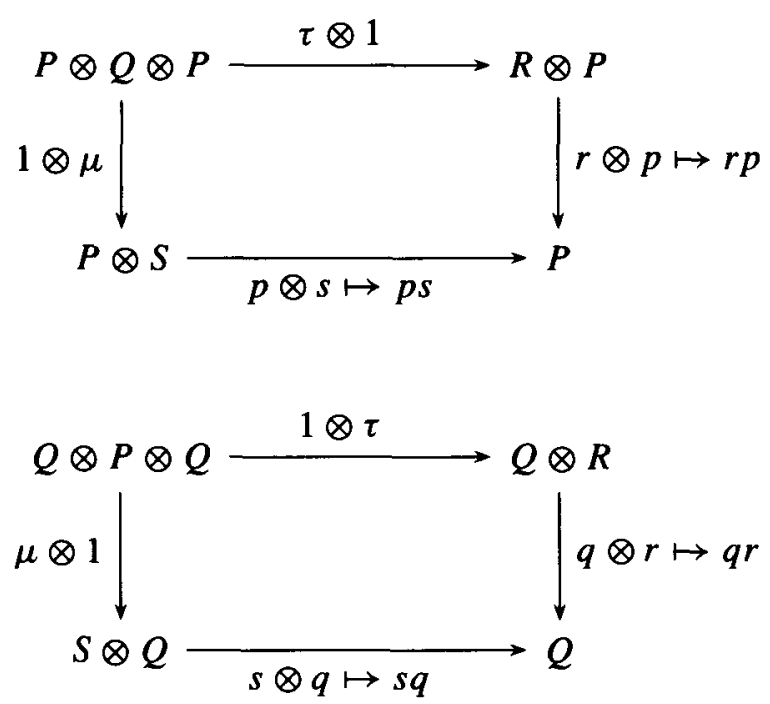


\section{Necessary conditions for equivalence}

We now use properties of equivalence to arrive at various necessary conditions. Throughout this section we shall assume that all semigroups have local units.

THEOREM 6.1. Let $R$ and $S$ be equivalent semigroups via inverse equivalences $G:$ FR-Act $\rightarrow$ FS-Act and $H:$ FS-Act $\rightarrow$ FR-Act.

Set $P=H\left({ }_{S} S\right)$ and $Q=G\left({ }_{R} R\right)$. Then $P$ and $Q$ are unitary biacts ${ }_{R} P_{S}$ and ${ }_{S} Q_{R}$ respectively such that

(1) ${ }_{R} P$ and ${ }_{S} Q$ are generators for FR-Act and FS-Act respectively;

(2) $R \cong R \otimes{ }_{R} \operatorname{End}_{S} Q, S \cong S \otimes{ }_{S} \operatorname{End}_{R} P$ as semigroups;

(3) $G \approx S \otimes{ }_{S} \operatorname{Hom}_{R}(P,-), H \approx R \otimes{ }_{R} \operatorname{Hom}_{S}(Q,-)$;

(4) ${ }_{S} Q_{R} \cong S \otimes{ }_{S} \operatorname{Hom}_{R}(P, R),{ }_{R} P S \cong R \otimes{ }_{R} \operatorname{Hom}_{S}(Q, S)$.

PROOF. (1) ${ }_{S} Q$ is naturally a right $\operatorname{End}_{S} Q$-act and $\operatorname{End}_{s} Q \cong \operatorname{End}_{R} R$, so ${ }_{s} Q$ is a right $\operatorname{End}_{R} R$-act. Now $\Theta: r \mapsto \rho(r)$ is a homomorphism of $R$ into $\operatorname{End}_{R} R$. Defining $q . r=q(r \Theta)=q G(\rho(r))$ we have that $Q$ is an $S$ - $R$-biact. Similarly, $P$ is an $R$ - $S$-biact.

Since ${ }_{R} R$ and ${ }_{S} S$ are generators, the same holds for ${ }_{S} Q$ and ${ }_{R} P$. In order to show that $Q_{R}$ is unitary, take an arbitrary element $q \in Q$. Since $Q=\bigcup\left\{G(R e): e^{2}=e \in R\right\}$, there is an idempotent $e \in R$ such that $q \in G(R e)$. Now $\rho(e) \in \operatorname{End}_{R} R$ and $\rho(e)$ acts as an identity on $R e$. Hence $G(\rho(e))$ acts as an identity on $G(R e)$ and we have $q e=q(G \rho(e))=q$. It is clear that $Q e=G(R e)$ is an indecomposable projective.

(2) Since $R \cong R \otimes_{R}$ End $_{R} R$ and $\operatorname{End}_{R} R \cong \operatorname{End}_{S} Q$, we may define multiplication on $R \otimes{ }_{R}$ End ${ }_{s} Q$ by $(a \otimes \xi)(c \otimes \omega)=a \xi^{\prime} \otimes c \cdot \omega$, where $\xi^{\prime}$ is the unique element of End $_{R} R$ corresponding to $\xi \in$ End $_{S} Q$. The multiplication is clearly well-defined and associative. Furthermore the map $a \otimes \xi^{\prime} \mapsto a \otimes \xi$ is an isomorphism of $R \otimes{ }_{R} \operatorname{End}_{R} R$ onto $R \otimes{ }_{R}$ End $_{S} Q$. Similarly $S \cong S \otimes{ }_{S} S \operatorname{End}_{R} P$.

(3) For ${ }_{R} M \in$ FR-Act we have that $G(M) \cong S \otimes{ }_{S} \operatorname{Hom}_{S}(S, G(M))$ by Lemma 4.1. Since $G$ and $H$ are inverse equivalences,

$$
\operatorname{Hom}_{S}(S, G(M)) \cong \operatorname{Hom}_{R}(H(S), M)=\operatorname{Hom}_{R}(P, M),
$$

and it is now easy to see that $G \approx S \otimes{ }_{S} \operatorname{Hom}_{R}(P,-)$. Similarly $H \approx R \otimes$ ${ }_{R} \operatorname{Hom}_{s}(Q,-)$.

(4) Let $\tilde{\Gamma}$ be the natural isomorphism such that $G \approx S \otimes{ }_{S} \operatorname{Hom}_{R}(P,-)$. Since $\tilde{\Gamma}$ is natural and $\rho(r) \in \operatorname{End}_{R} R$ for $r \in R$, we have that the following diagram 


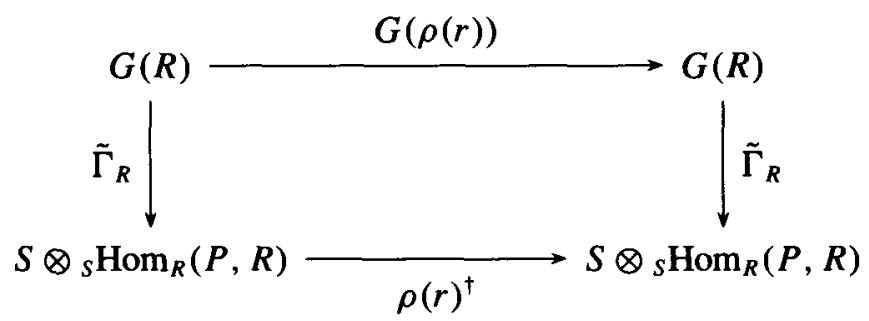

commutes, where

$$
\begin{gathered}
\rho(r)^{\dagger}=S \otimes{ }_{s} \operatorname{Hom}_{R}(P, \rho(r)): S \otimes{ }_{S} \operatorname{Hom}_{R}(P, R) \rightarrow S \otimes{ }_{s} \operatorname{Hom}_{R}(P, R) \\
s \otimes \omega \mapsto s \otimes \omega \rho(r)=s \otimes \omega \cdot r=(s \otimes \omega) r .
\end{gathered}
$$

By the commutativity of the diagram we have for $q \in Q$

$$
(q r) \tilde{\Gamma}_{R}=(q G(\rho(r))) \tilde{\Gamma}_{R}=\left(q \tilde{\Gamma}_{R}\right) \rho(r)^{\dagger}=\left(q \tilde{\Gamma}_{R}\right) r ;
$$

and it follows that $\tilde{\Gamma}_{R}$ is also a right $R$-isomorphism. Similarly we show that ${ }_{R} P_{S} \cong$ $R \otimes{ }_{R} \operatorname{Hom}_{S}(Q, S)$.

When $R$ and $S$ are monoids we have the following form of the necessary part of Knauer's main theorem.

COROLlARY 6.2. Let $R$ and $S$ be equivalent monoids via inverse equivalences $G:$ R-Act $\rightarrow$ S-Act and $H:$ S-Act $\rightarrow$ R-Act.

Set $P=H\left({ }_{S} S\right)$ and $Q=G\left({ }_{R} R\right)$. Then $P$ and $Q$ are unitary biacts ${ }_{R} P_{S}$ and ${ }_{S} Q_{R}$ respectively such that

(1) ${ }_{R} P$ and ${ }_{S} Q$ are generators for $\mathbf{R}$-Act and $\mathbf{S}$-Act respectively;

(2) $R \cong \operatorname{End}_{S} Q, S \cong \operatorname{End}_{R} P$ are semigroups;

(3) $G \approx \operatorname{Hom}_{R}(P,-), H \approx \operatorname{Hom}_{S}(Q,-)$;

(4) ${ }_{s} Q_{R} \cong \operatorname{Hom}_{R}(P, R),{ }_{R} P_{S} \cong \operatorname{Hom}_{S}(Q, S)$.

Moreover, there exists an idempotent $e$ in $R$ such that $S \cong e R e$ and $R=R e R$.

PROOF. It has already been noted that for a monoid $R, \mathbf{R}$-Act coincides with FR-Act. The conditions (2), (3) and (4) follow from the fact that for a monoid $R$ and a left $R$-act $M, R \otimes_{R} M \cong M$.

Since $S$ is a monoid, it is an indecomposable projective left $S$-act. It follows therefore that $P=H\left({ }_{s} S\right)$ is an indecomposable projective in R-Act. By Theorem 2.9 there exists an idempotent $e$ in $R$ such that ${ }_{R} P \cong{ }_{R} R e$, so that by (2) we have that $S \cong \operatorname{End}_{R} R e \cong e R e$. 
Let $r \in R$. We note that since $R$ is an indecomposable projective and $R e$ is a generator for $\mathbf{R}$-Act, there exists a surjective $\Omega \in \operatorname{Hom}_{R}(R e, R)$. It follows that $r=(s e) \Omega=s e(e \Omega) \in R e R$ for some $s e \in R e$.

\section{The Morita context associated with equivalence}

Equivalence is used in ring theory by drawing important conclusions from the surjectivity of $\tau$ and $\mu$ in an associated Morita context. In this section we shall show that if $R$ and $S$ are equivalent semigroups with local units, then we may define a Morita context such that $\tau$ and $\mu$ are surjective.

Let $R$ and $S$ be equivalent semigroups as in Theorem 6.1. We identify $S$ with $S \otimes$ ${ }_{s} \operatorname{Hom}_{R}(P, P)$ and $Q_{R}$ with $S \otimes{ }_{S} \operatorname{Hom}_{R}(P, R)$. Let $q=s \otimes \Phi \in S \otimes{ }_{s} \operatorname{Hom}_{R}(P, R)$ and $p \in P$, and consider the maps:

$$
\tau: P \otimes Q \rightarrow R, \quad p \otimes q \mapsto\langle p, q\rangle=p s \cdot \Phi
$$

and

$$
\mu: Q \otimes P \rightarrow S, \quad q \otimes p \mapsto[q, p]=s \otimes \delta,
$$

where $p^{\prime} \delta=\left(p^{\prime} \Phi\right) p$ for all $p^{\prime} \in P$. We note first that $\delta \in \operatorname{Hom}_{s}(P, P)$. For let $r \in R$ and $p^{\prime} \in P$. Then $\left(r p^{\prime}\right) \delta=\left(\left(r p^{\prime}\right) \Phi\right) p=r\left(p^{\prime} \Phi\right) p=r\left(\left(p^{\prime} \Phi\right) p\right)=r\left(p^{\prime} \delta\right)$.

It is an easy exercise to show that $\tau$ and $\mu$ are well-defined and are $R-R$ - and $S$-S-morphisms respectively.

LEMMA 7.1. $[q, p] q^{\prime}=q\left\langle p, q^{\prime}\right\rangle$ for all $q, q^{\prime} \in Q$ and all $p \in P$.

ProOF. Let $q=s \otimes \Phi$ and $q^{\prime}=u \otimes \Psi$ for $s, u \in S$ and $\Phi, \Psi \in \operatorname{Hom}_{R}(P, R)$. As above we write $[q, p]=\dot{s} \otimes \delta$.

Now $\operatorname{Hom}_{R}(P, P) \cong \operatorname{Hom}_{S}(S, S)$ and for each $\delta \in \operatorname{Hom}_{R}(P, P)$ we let $\delta^{\prime}$ be the corresponding element in $\operatorname{Hom}_{S}(S, S)$. Also $S \otimes \operatorname{Hom}_{S}(S, S)$ acts on $Q$ by $(a \otimes \xi) q=(a \xi) q$, and hence

$$
[q, p] q^{\prime}=\left(s \delta^{\prime}\right) u \otimes \Psi=e_{s} \otimes\left(s \delta^{\prime}\right) u \cdot \Psi .
$$

We also have that

$$
q\left\langle p, q^{\prime}\right\rangle=(s \otimes \Phi)\left\langle p, q^{\prime}\right\rangle=s \otimes \Phi \cdot\left\langle p, q^{\prime}\right\rangle=e_{s} \otimes s \cdot \Phi \cdot((p u) \Psi)
$$

where, since $\operatorname{Hom}_{R}(P, R)$ is a right $R$-act, we have $s \cdot \Phi((p u) \Psi) \in \operatorname{Hom}_{R}(P, R)$.

We show that $\left(s \delta^{\prime}\right) u \cdot \Psi=s \cdot \Phi \cdot((p u) \Psi)$. Let $p^{\prime \prime} \in P$. Then

$$
p^{\prime \prime}\left(\left(s \delta^{\prime}\right) u \cdot \Psi\right)=\left(p^{\prime \prime}\left(s \delta^{\prime}\right) u\right) \Psi \quad \text { and } \quad p^{\prime \prime}(s \cdot \Phi \cdot((p u) \Psi))=\left(\left(p^{\prime \prime} s\right) \Phi\right)((p u) \Psi)
$$


so if we can show that

$$
p^{\prime \prime}\left(s \delta^{\prime}\right)=\left(\left(p^{\prime \prime} s\right) \Phi\right) p
$$

then we are done.

Now $P$ is a right $\operatorname{Hom}_{R}(P, P)$-act, and therefore $p^{\prime \prime} \delta=p^{\prime \prime} \delta^{\prime}$, by definition of action by isomorphism. We have $p^{\prime \prime}\left(s \delta^{\prime}\right)=p^{\prime \prime} \rho\left(s \delta^{\prime}\right)$, where $\rho\left(s \delta^{\prime}\right) \in \operatorname{Hom}_{S}(S, S)$. But $\rho\left(s \delta^{\prime}\right)=s \cdot \delta^{\prime}$; hence

$$
p^{\prime \prime}\left(s \delta^{\prime}\right)=p^{\prime \prime} \rho\left(s \delta^{\prime}\right)=p^{\prime \prime}\left(s \cdot \delta^{\prime}\right)=p^{\prime \prime}(s \cdot \delta)=\left(p^{\prime \prime} s\right) \delta=\left(\left(p^{\prime \prime} s\right) \Phi\right) p
$$

where the last equality follows from the definition of $\delta$.

LEMMA 7.2. $p^{\prime}[q, p]=\left\langle p^{\prime}, q\right\rangle p$ for all $p, p^{\prime} \in P$ and all $q \in Q$.

ProOF. Let $q=s \otimes \Phi$. Then $\left\langle p^{\prime}, q\right\rangle p=\left(p^{\prime}(s \cdot \Phi)\right) p$ and $p^{\prime}[q, p]=p^{\prime}(s \otimes \delta)$, where $\delta$ is as in Lemma 7.1. Now

$$
p^{\prime}(s \otimes \delta)=p^{\prime}\left(s \otimes \delta^{\prime}\right)=p^{\prime}\left(s \delta^{\prime}\right)
$$

and from (\#) we have

$$
p^{\prime}\left(s \delta^{\prime}\right)=\left(\left(p^{\prime} s\right) \Phi\right) p=\left(p^{\prime}(s \cdot \Phi)\right) p
$$

The result follows.

The compatibility conditions proved above allow us to prove the following.

THEOREM 7.3. Let $R$ and $S$ be equivalent semigroups as in Theorem 6.1. We may identify $S$ with $S \otimes_{S} \operatorname{Hom}_{R}(P, P)$ and $Q_{R}$ with $S \otimes_{S} \operatorname{Hom}_{R}(P, R)$. Let $q=s \otimes \Phi \in Q$ and $p \in P$. Define the maps $\tau: P \otimes Q \rightarrow R$ by $p \otimes q \mapsto\langle p, q\rangle=p s \cdot \Phi$ and $\mu: Q \otimes P \rightarrow S$ by $q \otimes p \mapsto[q, p]=s \otimes \delta$, where $\delta: p^{\prime} \in P \mapsto\left(p^{\prime} \Phi\right) p \in P$. Then the six-tuple $\langle R, S, P, Q, \tau, \mu\rangle$ defines a Morita context. Furthermore $P \otimes{ }_{S} Q$ and $Q \otimes{ }_{R} P$ become semigroups if we put $\left(p_{1} \otimes q_{1}\right)\left(p_{2} \otimes q_{2}\right)=p_{1} \otimes s\left[q_{1}, p_{2}\right] q_{2}$ and $\left(q_{1} \otimes p_{1}\right)\left(q_{2} \otimes p_{2}\right)=q_{1} \otimes R_{R}\left\langle p_{1}, q_{2}\right\rangle p_{2}$ respectively. Moreover the maps $\tau$ and $\mu$ as defined above are surjective semigroup homomorphisms.

PROOF. We have already shown that $\langle R, S, P, Q, \tau, \mu\rangle$ defines a Morita context. It is easy to check that $P \otimes{ }_{s} Q$ and $Q \otimes_{R} P$ are in fact semigroups. Moreover,

$$
\begin{aligned}
& \left\{\left(p_{1} \otimes q_{1}\right)\left(p_{2} \otimes q_{2}\right)\right\} \tau=\left\{p_{1} \otimes\left[q_{1}, p_{2}\right] q_{2}\right\} \tau=\left\langle p_{1},\left[q_{1}, p_{2}\right] q_{2}\right\rangle \\
& =\left\langle\left[q_{1}, p_{2}\right], q_{2}\right\rangle=\left\langle p_{1}, q_{1}\right\rangle\left\langle p_{2}, q_{2}\right\rangle=\left(p_{1} \otimes q_{1}\right) \tau\left(p_{2} \otimes q_{2}\right) \tau
\end{aligned}
$$


Hence $\tau$ is a semigroup homomorphism. Similarly we may show that $\mu$ is also a semigroup homomorphism.

Since ${ }_{R} P$ is a generator it generates ${ }_{R} R$, and so every $r \in R$ can be written as $r=p \chi$, for some $p \in P$ and $\chi \in \operatorname{Hom}_{R}(P, R)$ depending on $r$. Since $P_{S}$ is also unitary, there exists an idempotent $f$ in $S$ such that $p=p f$.

Let $q=f \otimes \chi$. Then $p \otimes q \mapsto\langle p, q\rangle=p(f \cdot \chi)=p \chi=r$ and we have that $\tau$ is surjective.

To show $\mu$ is surjective let $g$ be an idempotent in $S$. Then $P g=H(S g)$ is an indecomposable projective left $R$-act, so there exists $e^{2}=e \in R$ and $\lambda \epsilon$ $\operatorname{Hom}_{R}(R e, P g)$ such that $\lambda$ is an isomorphism. Therefore we may write any $p \in P g$ as

$$
p=(r e) \lambda=r e(e \lambda)=r e \check{p} \text { where } \check{p}=e \lambda \in P g .
$$

Denote by $\check{q}$ the mapping which assigns to each $p \in P g$ the corresponding re. Then $\check{q}$ is clearly a well-defined left $R$-morphism from $P g$ to $R e \subseteq R$.

We now extend $\check{q}$ to the whole of $P$ by putting $p \check{q}=(p g) \check{q}$ and then by writing $q^{\prime}=g \otimes \check{q}$ we have for every $p \in P$,

$$
\left\langle p, q^{\prime}\right\rangle=p(g \cdot \check{q})=(p g) \check{q}=(p g)(g \cdot \check{q})=\left\langle p g, q^{\prime}\right\rangle .
$$

Moreover, we may rewrite $(1)$ as $p g=\langle p g, \check{q}\rangle \check{p}$, and we deduce that $p\left[q^{\prime}, \check{p}\right]=$ $\left\langle p, q^{\prime}\right\rangle \check{p}=p g$, that is, $p\{g \otimes \delta\}=p\left\{g \otimes \rho^{\prime}(g)\right\}$ where $\delta \in \operatorname{Hom}_{R}(P, P)$ is defined by $p^{\prime \prime} \delta=\left(p^{\prime \prime} \check{q}\right) \check{p}$ for all $p^{\prime \prime} \in P$, and $\rho^{\prime}(g)$ is the unique element of $\operatorname{Hom}_{R}(P, P)$ corresponding to $\rho(g) \in \operatorname{Hom}_{S}(S, S)$.

Now by definition of action by homomorphism, we have that for all $p \in P$, $p(g \cdot \delta)=p\left(g \cdot \rho^{\prime}(g)\right)$, that is, $g \cdot \delta=g \cdot \rho^{\prime}(g)$.

If $s \in S$ and $p \in P$ are arbitrary, then $s=g s$ for some $g^{2}=g \in S$, and

$$
p s=p\left(g \cdot \rho^{\prime}(s)\right)=p\left(g \cdot \rho^{\prime}(g)\left(\rho^{\prime}(s)\right)\right)=(p(g \cdot \delta)) \rho^{\prime}(s)
$$

where $\rho^{\prime}(s)$ is the unique element of $\operatorname{Hom}_{R}(P, P)$ corresponding to $\rho(s) \epsilon$ $\operatorname{Hom}_{S}(S, S)$. It therefore follows that $g \cdot \rho^{\prime}(s)=g \delta \cdot \rho^{\prime}(s)$ and so $g \otimes g \cdot \rho^{\prime}(s)=$ $g \otimes g \cdot \delta \rho^{\prime}(s)$, and we have $\left(q^{\prime} \otimes \check{p} s\right) \mu=g \otimes g \delta \cdot \rho^{\prime}(s)=g \otimes g \cdot \rho^{\prime}(s)$, which is the element of $S \otimes \operatorname{Hom}_{R}(P, P)$ corresponding to $s \in S$.

\section{Morita context and sufficient conditions for equivalence}

DEFINITION. We say that a Morita context $\left\langle R, S,{ }_{R} P_{S},{ }_{S} Q_{R},\langle\rangle,,[],\right\rangle$ is unitary if $R$ and $S$ are semigroups with local units, ${ }_{R} P \in$ FR-Act, $s Q \in$ FS-Act, and the biacts ${ }_{R} P_{S}$ and ${ }_{S} Q_{R}$ are unitary. 
As in the module case, we shall now show that the existence of a unitary Morita context with $\langle$,$\rangle and [, ] surjective, is sufficient for the semigroups R$ and $S$ to be Morita equivalent. Moreover, it is also possible to recover a great deal of information from a Morita context. Firstly, we give the following lemma.

LEMMA 8.1. Let $\left\langle R, S,{ }_{R} P_{S},{ }_{s} Q_{R},\langle\rangle,,[],\right\rangle$ be a unitary Morita context with $\langle$, and $[$,$] surjective. Then \langle$,$\rangle and [$,$] are also injective.$

PROOF. Let [, ] $s Q \otimes_{R} P_{S} \rightarrow S$ be surjective and suppose that $[q, p]=\left[q^{\prime}, p^{\prime}\right]$. Let $e$ and $f$ be idempotents in $S$ such that $e q=q$ and $p^{\prime} f=p^{\prime}$, let $\left[q^{\prime \prime}, p^{\prime \prime}\right]=e$ and $\left[q^{\prime \prime \prime}, p^{\prime \prime \prime}\right]=f$. Then

$$
\left[q^{\prime \prime}, p^{\prime \prime}\right]\left[q^{\prime}, p^{\prime}\right]=\left[q^{\prime \prime}, p^{\prime \prime}\right][q, p]=[q, p]\left[q^{\prime}, p^{\prime}\right]
$$

Now, $q \otimes p=\left[q^{\prime \prime}, p^{\prime \prime}\right] q \otimes p=q^{\prime \prime} \otimes\left\langle p^{\prime \prime}, q\right\rangle p=q^{\prime \prime} \otimes p^{\prime \prime}\left[q^{\prime}, p^{\prime}\right]=\left[q^{\prime \prime}, p^{\prime \prime}\right] q^{\prime} \otimes p^{\prime}=$ $\left[q^{\prime \prime}, p^{\prime \prime}\right] q^{\prime} \otimes p^{\prime}\left[q^{\prime \prime \prime}, p^{\prime \prime \prime}\right]=q^{\prime} \otimes p^{\prime}$. Similarly, we may show that $\langle$,$\rangle is also injective.$

The proof of the above lemma allows us to give an alternative characterisation of the category FS-Act.

LEMMA 8.2. A unitary $S$-act $M$ is in FS-Act if and only if $S \otimes M \cong M$.

ProOF. Suppose that $M \in$ FS-Act. Then by definition $S \otimes \operatorname{Hom}_{S}(S, M) \cong M$. By the proof in the above lemma, $S \otimes S \cong S$, and it follows that $S \otimes M \cong M$. The converse follows from Lemma 4.8 .

THEOREM 8.3. Let $\left\langle R, S,{ }_{R} P_{S},{ }_{s} Q_{R},\langle\rangle,,[],\right\rangle$ be a unitary Morita context with $\langle$,$\rangle :$ $P \otimes Q \rightarrow R$ and [,] $: Q \otimes P \rightarrow S$ surjective. Then the following hold:

(1) The categories FR-Act and FS-Act are equivalent via the functors ${ }_{s} Q_{R}$ : FR-Act $\rightarrow$ FS-Act and ${ }_{R} P \otimes_{s}:$ FS-Act $\rightarrow$ FR-Act.

(2) ${ }_{R} P$ and ${ }_{S} Q$ are generators for FR-Act and FS-Act respectively.

(3) $S \otimes{ }_{S} \operatorname{Hom}_{R}(P, R) \cong{ }_{S} Q_{R}$ and $R \otimes{ }_{R} \operatorname{Hom}_{S}(Q, S) \cong{ }_{R} P_{S}$ as biacts.

(4) $R \cong R \otimes{ }_{R}$ End $_{S} Q$ and $S \cong S \otimes{ }_{S}$ End $_{R} P$ as semigroups.

ProOF. (1) Let $M \in$ FS-Act. Then

$$
{ }_{S} Q \otimes{ }_{R}\left(P \otimes{ }_{S} M\right) \cong\left({ }_{s} Q \otimes{ }_{R} P\right) \otimes{ }_{S} M \cong S \otimes M \cong{ }_{S} M
$$

Similarly if $N \in$ FR-Act, then

$$
{ }_{R} P \otimes{ }_{S} Q \otimes{ }_{R} N \cong{ }_{R} N
$$


(2) We note first that for each $q \in Q$ we may define the map $\langle, q\rangle:{ }_{R} P \rightarrow{ }_{R} R$ by $p \mapsto\langle p, q\rangle$. Since $\langle$,$\rangle is a left R$-morphism, it follows that $\langle, q\rangle$ is also a left $R$-morphism. We have already noted that ${ }_{R} R$ is a generator for FR-Act, and it follows from the surjectivity of $\langle$,$\rangle that { }_{R} P$ is also a generator for FR-Act.

Similarly for each $p \in P$ we may define the left $S$-morphism [, $p]:{ }_{s} Q \rightarrow{ }_{s} S$ by $q \mapsto[q, p]$

(3) For each $q \in Q$ and $p \in P$, let $\langle, q\rangle$ and [, $p$ ] be defined as in (2). We have noted that $[, p] \in \operatorname{Hom}_{S}(Q, S)$. Furthermore, for any $n \in N$ where $N \in$ FS-Act the map [, $p] . n \in \operatorname{Hom}_{S}(Q, N)$. Similar remarks apply to the map $\langle, q\rangle$.

We define a map $\Phi:{ }_{R} P_{S} \rightarrow R \otimes{ }_{R} \operatorname{Hom}_{S}(Q, S)$ by $p \mapsto e \otimes[, p]$, where $e p=p$. We claim that $\Phi$ is well-defined. Suppose that $e^{\prime}$ is an idempotent such that $e^{\prime} p=p$. Let $f$ be an idempotent in $S$ such that $p f=f$, and let $f=\left[q^{\prime}, p^{\prime}\right]$. Then

$$
\begin{aligned}
e \otimes[, p] & =e \otimes[, p]\left[q^{\prime}, p^{\prime}\right]=e \otimes\left[,\left\langle p, q^{\prime}\right\rangle p^{\prime}\right] \\
& =e \otimes\left\langle p, q^{\prime}\right\rangle \cdot\left[, p^{\prime}\right]=\left\langle p, q^{\prime}\right\rangle \otimes\left[, p^{\prime}\right]=e^{\prime} \otimes[, p] .
\end{aligned}
$$

It is easy to see that $\Phi$ is an $R$-S-biact morphism. We claim that $\Phi$ is also surjective. Let $r \otimes \alpha \in R \otimes{ }_{R} \operatorname{Hom}_{S}(Q, S)$ and let $r=\langle p, q\rangle$. Now, $q \alpha \in S$ and

$$
p(q \alpha) \mapsto e \otimes[, p(q \alpha)] \text { where } e(p(q \alpha))=p(q \alpha) \text {. }
$$

Let $q^{\prime} \in Q$. Then

$$
q^{\prime}[, p(q \alpha)]=\left[q^{\prime}, p(q \alpha)\right]=\left[q^{\prime}, p\right](q \alpha)=\left(q^{\prime}\langle p, q\rangle\right) \alpha=q^{\prime}(r . \alpha)
$$

and the result follows. It is easy to see that the map $\Phi^{\prime}: R \otimes{ }_{R} \operatorname{Hom}_{S}(Q, S) \rightarrow{ }_{R} P_{S}$ defined by $r \otimes \alpha \mapsto p(q \alpha)$, where $r=\langle p, q\rangle$, is well-defined and the inverse of $\Phi$.

(4) $R \otimes{ }_{R}$ End $_{S} Q$ is indeed a semigroup if we define multiplication by

$$
(r \otimes \alpha)\left(r^{\prime} \otimes \alpha^{\prime}\right)=r \otimes \alpha r^{\prime} \cdot \alpha^{\prime} .
$$

We identify $R$ with $P \otimes Q$ and define maps $R \otimes_{R}$ End $_{s} Q \rightarrow P \otimes Q$ by $r \otimes \alpha \mapsto p \otimes q \alpha$, where $r=\langle p, q\rangle$, and $P \otimes Q \rightarrow R \otimes{ }_{R}$ End $_{S} Q$ by $p \otimes q \mapsto e \otimes \rho(\langle p, q\rangle)$. It is easy to check that the maps are mutually inverse semigroup morphisms.

\section{Semigroups of the form $S=S e S$, $e$ an idempotent}

We now make use of our main theorem to characterise those semigroups with local units which are Morita equivalent to a monoid. We find that semigroups of the form $S=S e S$, for $e$ an idempotent are the only such semigroups. When $S$ is a monoid satisfying this condition we retrieve Knauer's main result. We also state some wellknown results of Hotzel (1976), and deduce the Rees theorem for completely 0-simple semigroups. 
THEOREM 9.1. A semigroup $S$ with local units is Morita equivalent to a monoid if and only if there exists $e^{2}=e \in S$ such that $S=S e S$. If this is the case then $S$ is Morita equivalent to the monoid $e$ Se.

PROOF. Suppose that an idempotent as described in the statement exists and consider the six-tuple:

$$
\left\langle S, \operatorname{Hom}_{S}(\mathrm{Se}, \mathrm{Se}), P=\mathrm{Se}, Q=\operatorname{Hom}_{S}(\mathrm{Se}, S) S, \tau, \mu\right\rangle
$$

where for $p \in S e$ and $q \in \operatorname{Hom}_{S}(\operatorname{Se}, S) S$, we define:

$$
\begin{gathered}
\tau: S e \otimes e S_{e} \operatorname{Hom}_{S}(S e, S) \rightarrow S, \quad p \otimes q \mapsto\langle p, q\rangle=p q ; \quad \text { and } \\
\mu: \operatorname{Hom}_{S}(S e, S) \otimes S_{S e} \rightarrow \operatorname{Hom}_{s}(S e, S e), \\
q \otimes p \mapsto[q, p]=\langle, q\rangle p: p^{\prime} \mapsto\left\langle p^{\prime}, q\right\rangle p,
\end{gathered}
$$

for $p^{\prime} \in S e$.

Clearly $\tau$ is an $S$-S-morphism and $\mu$ a $\operatorname{Hom}_{S}(S e, S e)-H_{S o m}(S e, S e)$-morphism. We note that for any $p \in S e$ and $q, q^{\prime} \in \operatorname{Hom}_{S}(S e, S) S, q\left\langle p, q^{\prime}\right\rangle$ and $[q, p] q^{\prime}$ belong to $\mathrm{Hom}_{s}(\mathrm{Se}, S) S$. For $p^{\prime} \in P$,

$$
\begin{aligned}
p^{\prime}\left(q\left\langle p, q^{\prime}\right\rangle\right) & =\left\langle p^{\prime}, q\right\rangle\left\langle p, q^{\prime}\right\rangle\left\langle\left\langle p^{\prime}, q\right\rangle p, q^{\prime}\right\rangle \\
& =\left\langle p^{\prime}[q, p], q^{\prime}\right\rangle=\left\langle p^{\prime},[q, p] q^{\prime}\right\rangle .
\end{aligned}
$$

It therefore follows that $q\left\langle p, q^{\prime}\right\rangle=[q, p] q^{\prime}$. Also from the definition of $\tau$ and $\mu$ we have that $\langle p, q\rangle p^{\prime}=p\left[q, p^{\prime}\right]$. Furthermore, since $e S e \cong \operatorname{Hom}_{S}(\mathrm{Se}, \mathrm{Se})$ and $e S \cong \operatorname{Hom}_{S}(\mathrm{Se}, S) S$, we have that the above six-tuple defines a unitary Morita context.

Let $M \in$ US-Act. For $m \in M$ the map

$$
\rho=\coprod_{m \in M} \rho(m): \coprod_{m \in M} P \rightarrow M
$$

is a surjective left $S$-morphism, for $\operatorname{Im} \rho=\operatorname{Se} M=\operatorname{Se}(S M)=S M=M$. Therefore $P=S e$ is a projective indecomposable generator for US-Act.

Since $P$ is a generator for US-Act and $S \in$ US-Act, it follows that for any $s \in S$ there exists $p \in P$ and $q \in \operatorname{Hom}_{S}(P, S)$, such that $p q=s$ and we have that $\tau$ is surjective.

To show that $\mu$ is surjective, let $\Phi \in \operatorname{Hom}_{S}(S e, S e)$. Then $e \Phi \in P=S e$. Moreover, $\rho(e) \in \operatorname{Hom}_{S}(P, S) S$, and we have that

$$
\rho(e) \otimes e \Phi \mapsto\langle, \rho(e)\rangle(e \Phi): s e \in S e \mapsto(s e)(e \Phi)=(s e) \Phi .
$$

By Theorem 8.6 it follows that $S$ is Morita equivalent to $\operatorname{Hom}_{S}(\mathrm{Se}, \mathrm{Se}) \cong e S e$. 
Conversely, suppose $S$ is Morita equivalent to a monoid $R$. Then there exists an inverse equivalence $(G, H)$, such that

$$
G: \text { FS-Act } \rightarrow \text { FR-Act and } H: \text { FR-Act } \rightarrow \text { FS-Act. }
$$

Since ${ }_{R} R$ is an indecomposable projective generator, we have that $P=H\left({ }_{R} R\right)$ is also an indecomposable projective generator in FS-Act. It follows that there exists an $e^{2}=e \in S$ and $\Omega \in \operatorname{Hom}_{S}(S e, P)$ such that $\Omega$ is surjective. Thus $S e$ is also a generator for FS-Act.

Let $x \in S$. Then $x=x f$ for some $f^{2}=f \in S$. Since $S f$ is an indecomposable projective there exists a surjective $\bar{\Omega} \in \operatorname{Hom}_{S}(\operatorname{Se}, S f)$ and we have that for some $s e \in \operatorname{Se}, x=x f=(s e) \bar{\Omega}=s e(e \bar{\Omega}) \in \operatorname{SeS}$.

Since a monoid is a semigroup with local units, we deduce the following from the above theorem and Corollary 6.2:

COROLlARY 9.2. The monoids $A$ and $B$ are Morita equivalent if and only if there exists an idempotent $e \in A$ such that $A=A e A$ and $B \cong e A e$.

If $A$ is a monoid then FA-Act is in fact A-Act as studied by Knauer. Corollary 9.2 is therefore just Knauer's theorem on Morita equivalence.

We also have the following from Knauer (1972):

COROLlary 9.3. Morita equivalence of the monoids $A$ and $B$ implies that $A$ and $B$ are isomorphic monoids in any of the following cases:

(1) A is a commutative monoid.

(2) In A there is at most one element of finite order.

(3) The identity element $e$ of $A$ is externally adjoined, that is, from $a b=e, a, b \in A$, it follows that $a=b=e$.

(4) The monoid $A$ is a group.

\section{Morita equivalence and Rees Theorem}

DEFINITION. A semigroup $S$ without zero is called simple if it has no proper ideals. A semigroup with zero is called 0-simple if (1) $\{0\}$ and $S$ are the only ideals, and (2) $S^{2} \neq\{0\}$.

We have the following well-known characterisation of 0-simple semigroups in terms of elements.

LEMMA 9.4. A semigroup $S$ with zero is 0-simple if and only if $S a S=S$ for every a in $S \backslash\{0\}$. 
DEFINITION. A 0-simple semigroup is said to be completely 0 -simple if every nonempty set either of $L$-classes or of $R$-classes possesses a minimal member, where $L$ and $R$ are the Green's relations.

It is well-known that a 0 -simple semigroup is completely 0 -simple if and only if it contains a primitive idempotent.

The following construction of completely 0 -simple semigroups is due to Rees (1940).

Let $G$ be a group with identity element $e$, and let $I, \Lambda$ be non-empty sets. Let $P=\left(p_{\lambda i}\right)$ be a $\Lambda \times I$ matrix with entries in the 0 -group $G^{0}(=G U\{0\})$, and suppose that $P$ is regular, in the sense that no row or column consists entirely of zeros. Let $S=(G \times I \times \Lambda) \cup\{0\}$ and define a composition on $S$ by

$$
\begin{aligned}
(a, i, \lambda)(b, j, \mu) & =\left\{\begin{array}{lll}
\left(a p_{\lambda j} b, i, \mu\right) & \text { if } & p_{\lambda_{j}} \neq 0 \\
0 & \text { if } & p_{\lambda j}=0 .
\end{array}\right. \\
(a, i, \lambda) 0 & =0(a, i, \lambda)=00=0 .
\end{aligned}
$$

THEOREM 9.5. [Rees (1940)] $S$ as defined above is a completely 0-simple semigroup. Conversely, every completely 0-simple semigroup is isomorphic to one constructed in this way.

We now proceed to recover the Rees theorem. Firstly, from Lemma 8.1 we have:

COROLLARY 9.6. If $S$ is a completely 0-simple semigroup then $\tau$ and $\mu$ in Theorem 9.1 are injective.

We have almost proved the Rees theorem for completely 0 -simple semigroups. To deduce the Rees theorem from our work we use an alternative description of Rees matrix semigroups due to Hotzel (1976), which we now describe.

Let $D$ be a monoid with 0 and let ${ }_{D} M$ be a free unital $D$-act over the set $\left\{u_{\lambda}: \lambda \in \Lambda\right\}$ (we suppose that $\lambda \mapsto u_{\lambda}$ is one to one), so that ${ }_{D} M=\bigsqcup_{\lambda \in \Lambda} D u_{\lambda}$ (the 0-disjoint union of the subacts $D u_{\lambda}$ of ${ }_{D} M$ ) and $d \mapsto d u_{\lambda}$ is a $D$-isomorphism between ${ }_{D} D$ and $D u_{\lambda}$ for all $\lambda \in \Lambda$. Any $m \in M$ can be represented by a row-monomial row vector $m^{\prime}$ over D:

$$
m^{\prime}=\left(d_{\lambda}\right)_{\lambda \in \Lambda} \quad \text { with } \quad d_{\lambda}=g \quad \text { if } \quad m=g u_{\lambda}, \quad \text { and } \quad d_{\lambda}=0 \text { otherwise. }
$$

Any $D$-endomorphism $\rho$ can be represented by a row-monomial $\Lambda \times \Lambda$ matrix $\rho^{\prime}$ over $D$ :

$$
\rho^{\prime}=\left(r_{\lambda \mu}\right)_{\lambda, \mu \in \Lambda} \quad \text { with } \quad r_{\lambda \mu}=g \quad \text { if } \quad u_{\lambda} \rho=g u_{\mu}, \quad \text { and } \quad r_{\lambda \mu}=0 \text { otherwise. }
$$

Correspondingly, if $N_{D}$ is a free unital $D$-act with basis $\left\{v_{i}: i \in I\right\}$ then any $n \in N$ can be represented by a monomial column vector $n^{\prime}$ over $D$. 
Any $D$-endomorphism $\sigma$ of $N_{D}$ can be represented by a column monomial $I \times I$ matrix over $D$. Moreover a bilinear mapping [, ] from ${ }_{D} M \times N_{D}$ to ${ }_{D} D_{D}$ can be represented by a $\Lambda \times I$-sandwich matrix

$$
P=\left(\left[u_{\lambda}, v_{i}\right]\right)_{\lambda \in \Lambda, i \in I} .
$$

Now every element $n \otimes m$ of $N \otimes{ }_{D} M$ corresponds to the Rees matrix

$$
(n \otimes m)^{\dagger}=\left(d_{i \lambda}\right)_{i \in I, \lambda \in \Lambda},
$$

with $d_{i \lambda}=g$ if $n \otimes m=v_{i} g \otimes u_{\lambda}$ and $d_{i \lambda}=0$ otherwise. It can be shown that the mapping $n \otimes m \mapsto(n \otimes m)^{\dagger}$ is well-defined and bijective. If we define multiplication on $N \otimes M$ by

$$
(n \otimes m)\left(n_{1} \otimes m_{1}\right)=n \otimes\left[m, n_{1}\right] m_{1}
$$

we have $\left(v_{i} g \otimes u_{\lambda}\right)\left(v_{j} h \otimes u_{\mu}\right)=v_{i} g\left[u_{\lambda}, v_{j}\right] h \otimes u_{\mu}$. In $N \otimes{ }_{D} M$ we have

$$
\left(\left(v_{i} g \otimes u_{\lambda}\right)\left(v_{j} h \otimes u_{\mu}\right)\right)^{\dagger}=\left(v_{i} g \otimes u_{\mu}\right)^{\dagger} P\left(v_{j} h \otimes u_{\mu}\right)^{\dagger} .
$$

Thus $N \otimes{ }_{D} M$ is just a coordinate free version of the Rees matrix semigroup over $D$ with sandwich matrix $P$. Clearly, any Rees matrix semigroup over $D$ can be so obtained.

We also have the following lemma in Hotzel (1976):

LEMMA 9.7. Suppose that $e$ is an idempotent of $S$ and $S$ is a 0 -disjoint union of left ideals isomorphic with $\mathrm{Se}$. Then ${ }_{e} \mathrm{Se}_{e} \mathrm{~S}$ is a free unital eSe-act.

From Theorem 9.1 and Corollary 9.6 we have that if $S$ is a completely 0 -simple semigroup then $S \cong S e \otimes_{e s e} e S$. It is a well-known fact that a completely 0 -simple semigroup is a 0 -disjoint union of right ideals isomorphic with $e S$. It is also wellknown that $e S e$ is a group with zero. Therefore by the above remarks we have the Rees theorem for completely 0 -simple semigroups.

We have also proved half of

THEOREM 9.8. A regular semigroup $S$ with zero is completely 0 -simple if and only if $S$ is Morita equivalent to a group $G$ with zero.

ProOF. Suppose that $S$ is Morita equivalent to a group $G$ with 0 . Then by Theorem 9.1 there exists an idempotent $e$ in $S$ such that the monoid $e S e$ is Morita equivalent to $S$, hence also to $G$. Let $F: \mathbf{e S e}^{\mathbf{0}}$-Act $\rightarrow \mathbf{G}^{\mathbf{0}}$-Act and $H: \mathbf{G}^{\mathbf{0}}$-Act $\rightarrow \mathbf{e S e}^{\mathbf{0}}$-Act be inverse equivalences. Since $e S e$ is an indecomposable projective generator in $\mathrm{eSe}^{0}$-Act, we have that $P=F(e S e)$ is also an indecomposable projective generator in $\mathbf{G}^{0}$-Act. Thus there exists an idempotent $g$ in $G$ such that $P \cong G g$. The idempotent 
$g$ cannot be zero, for then $P$ would not be a generator. Hence $P \cong{ }_{G} G$ and it follows by Corollary 6.2 that $e S e \cong \operatorname{End}_{G} P \cong \operatorname{End}_{G} G \cong G$. Let $f$ be an idempotent in $S$ such that $f \leq e$. Then $f=e f e$ is an idempotent in the group $e S e$ and so $f$ is either 0 or $e$. Thus $S$ contains a primitive idempotent.

Let $a$ be a non-zero element of $S$. Since $S$ is regular, there exists an idempotent $g$ in $S$ such that $S a=S g$. We shall show that $S=S a S$. Let $x \in S$. Then $x=x f$ for some idempotent $f$ in $S$. Since $S g$ and $S f$ are both indecomposable and projective and all indecomposable projectives in $\mathbf{F G}^{\mathbf{0}}$-Act are isomorphic to $G$ we have that $S f$ and $S g$ are isomorphic left $S$-acts. Let $\Omega: S g \rightarrow S f$ be an isomorphism. Then there exist $s$ and $t$ in $S$ such that $x=x f=(s g) \Omega=s g(g \Omega)=t a(g \Omega) \in S a S$.

Given a group $G$ with 0 , we can construct the Rees matrix semigroup $S=M^{0}[G]$. By Rees' theorem $S$ is a completely 0 -simple semigroup and $G$ is isomorphic to $e S e$, for each non-zero idempotent $e$ of $S$. By Theorem $9.1 S$ is Morita equivalent to $e S e$ and so also to $G$.

\section{Bisimple semigroups and Morita equivalence}

The following is well-known.

PROPOSITION 9.9. The idempotent elements $e, f$ of a semigroup $S$ are D-related if and only if the left ideals $S e$ and $S f$ are isomorphic as left $S$-acts, where $D$ is the Green's relation.

We also have the following from Allen (1991).

LEMMA 9.10. If $S$ is a regular semigroup and if $e$ is any idempotent of $S$, then eSe is a regular subsemigroup of $S$.

THEOREM 9.11. A regular semigroup $S$ with zero is bisimple if and only if $S$ is Morita equivalent to a regular bisimple monoid with zero.

PROOF. Suppose that $S$ is Morita equivalent to a regular bisimple monoid $A$ with 0. Since all indecomposable projectives are isomorphic in $\mathbf{F A}^{\mathbf{0}}$-Act, we have that all indecomposable projectives are isomorphic in $\mathbf{F S}^{\mathbf{0}}$-Act. That is, $S e$ and $S f$ are isomorphic for all non-zero idempotents $e, f$ in $S$.

Conversely let $S$ be a regular bisimple semigroup with 0 and let $e$ be an idempotent in $S$ such that $S=S e S$. By Theorem 9.1, $S$ is Morita equivalent to the monoid $e S e$ (= $A$ ). Let $f, g$ be idempotents in $A$. Then $A f$ and $A g$ are indecomposable projectives in $\mathbf{F A}^{\mathbf{0}}$-Act. These must be isomorphic since all indecomposable projectives are isomorphic in $\mathbf{F S}^{\mathbf{0}}$-Act. It follows that $\boldsymbol{A}$ is a regular bisimple monoid with zero. 
COROLLARY 9.12. A regular semigroup $S$ is bisimple if and only if $S$ is Morita equivalent to a regular bisimple monoid.

The fundamental regular four spiral semigroup $S_{P 4}$ is an idempotent generated bisimple semigroup which is not completely simple. Formally, let $X=\{a, b, c, d\}$, let $\Delta_{0}=\left\{\left(a, a^{2}\right),\left(b, b^{2}\right),\left(c, c^{2}\right),\left(d, d^{2}\right),(a, b a),(b, a b),(b, b c),(c, c b),(c, d c)\right.$, $(d, c d),(d, d a)\}$, let $\Delta$ be the congruence generated by $\Delta_{0}$ on the free semigroup $F_{X}$ and let $S_{P 4}=F_{X} / \Delta$.

From Byleen, Meakin and Pastijn (1978), we know that $S_{P 4}$ is a bisimple regular semigroup generated by idempotents $a, b, c, d$ in which $a S_{P_{4}} a$ is isomorphic to the bicyclic semigroup. Clearly $S_{P 4}$ is simple and it follows that $S_{P 4}$ is Morita equivalent to the monoid $a S_{P 4} a$.

\section{Acknowledgements}

The research for this paper was done while the author was supported by the Science and Engineering Council in the form of a Research Studentship. The author would like to thank Dr. John Fountain for his invaluable help during this period.

\section{References}

[1] G. D. Abrams (1983), 'Morita equivalence for rings with local units', Comm. Algebra 11, 801-837.

[2] D. Allen (1991), 'A structure theory for finite regular semigroups', in: Monoids and Semigroups with Applications (ed. J. Rhodes) (World Scientific, Singapore) pp. 403-423.

[3] F. W. Anderson and K. R. Fuller (1974), Rings and categories of modules, Graduate Texts in Mathematics 13 (Springer, Berlin).

[4] P. N. Anh and L. Marki (1987), 'Morita equivalence for rings without identity', Tsukuba J. Math. 11, 1-16.

[5] B. Banaschewski (1972), 'Functors into the category of $M$-sets', Abh. Math. Sem. Univ. Hamburg 8, 49-64.

[6] J. M. Barga and E. G-Rodeja F (1980), 'Morita equivalence of monoids', Semigroup Forum 19, 101-106.

[7] K. Byleen, J. Meakin and F. Pastijn (1978), 'The fundamental four spiral semigroup', J. Algebra $54,6-26$.

[8] E. Hotzel (1976), 'Dual D-operands and the Rees Theorem', in: Algebraic theory of semigroups, Colloq. Math. Soc. János Bolyai 20.

[9] J. M. Howie (1976), An introduction to semigroup theory (Academic Press, London).

[10] N. Jacobson (1980), Basic Algebra 2 (Freeman, San Francisco).

[11] U. Knauer (1972), 'Projectivity of acts and morita equivalence of monoids', Semigroup Forum 3 , 359-370.

[12] U. Knauer and P. Normak (1990), 'Morita duality of monoids', Semigroup Forum 40, 39-57.

[13] B. Mitchell (1965), Theory of categories (Academic Press, London). 
[14] K. Morita (1961), 'Category-isomorphism and endomorphism rings of modules', Trans. Amer. Math. Soc. 103, 451-469.

[15] D. Rees (1940), ‘On semi-groups', Proc. Cambridge Phil. Soc. 36, 387-400.

Department of Mathematics

University of York

Heslington

York Y01 5DD 